\title{
A Respiration-Coupled Rhythm in the Rat Hippocampus Independent of Theta and Slow Oscillations
}

\author{
๑DAndré L. V. Lockmann, Diego A. Laplagne, Richardson N. Leão, and @Adriano B. L. Tort \\ Brain Institute, Federal University of Rio Grande do Norte, RN 59056-450, Brazil
}

During slow-wave sleep and deep anesthesia, the rat hippocampus displays a slow oscillation (SO) that follows "up-and-down" state transitions in the neocortex. There has been recent debate as to whether this local field potential (LFP) rhythm reflects internal processing or entrains with respiratory inputs. To solve this issue, here we have concomitantly recorded respiration along with hippocampal, neocortical, and olfactory bulb (OB) LFPs in rats anesthetized with urethane. During the course of anesthesia, LFPs transitioned between activity states characterized by the emergence of different oscillations. By jointly analyzing multisite LFPs and respiratory cycles, we could distinguish three types of low-frequency hippocampal oscillations: (1) SO, which coupled to neocortical up-and-down transitions; (2) theta, which phase-reversed across hippocampal layers and was largest at the fissure; and (3) a low-frequency rhythm with largest amplitude in the dentate gyrus, which coupled to respiration-entrained oscillations in $\mathrm{OB}$ and to respiration itself. In contrast, neither theta nor $\mathrm{SO}$ coupled to respiration. The hippocampal respiration-coupled rhythm and $\mathrm{SO}$ had frequency $<1.5 \mathrm{~Hz}$, whereas theta tended to be faster $(>3 \mathrm{~Hz})$. Tracheotomy abolished hippocampal respiration-coupled rhythm, which was restored by rhythmic delivery of air puffs into the nasal cavity. These results solve the apparent contradictions among previous studies by demonstrating that the rat hippocampus produces multiple types of low-frequency oscillations. Because they synchronize with different brain circuits, however, we postulate that each activity pattern plays a unique role in information processing.

Key words: hippocampal rhythms; LFP; olfactory bulb; respiration; slow oscillation; theta

Significance Statement

The rat hippocampus exhibits a large-amplitude slow oscillation $(<1.5 \mathrm{~Hz})$ during deep sleep and anesthesia. It is currently debated whether this rhythm reflects internal processing with the neocortex or entrainment by external inputs from rhythmic nasal respiration, which has similar frequency. Here we reconcile previous studies by showing that the hippocampus can actually produce two low-frequency rhythms at nearby frequencies: one that indeed couples to respiration and another that is coupled to the neocortex. We further show that the respiration-coupled rhythm differs from theta oscillations. The results support a role for brain oscillations in connecting distant brain regions, and posit the respiratory cycle as an important reference for neuronal communication between olfactory and memory networks.

\section{Introduction}

Local field potentials (LFPs) in the rodent hippocampus display oscillations at multiple frequencies, which have different generating mechanisms and behavioral/cognitive correlates (Buzsáki and Draguhn, 2004). Hippocampal subregions synchronize with other areas in a frequency-specific and state-dependent fashion

Received Sept. 13, 2015; revised March 13, 2016; accepted April 5, 2016.

Author contributions: A.L.V.L. and A.B.L.T. designed research; A.L.V.L. and D.A.L. performed research;D.A.L. and R.N.L. contributed unpublished reagents/analytic tools; A.L.V.L. and A.B.L.T. analyzed data; A.L.V.L. and A.B.L.T. wrote the paper.

This work was supported by Conselho Nacional de Desenvolvimento Científico e Tecnológico and Coordenação de Aperfeiçoamento de Pessoal de Nível Superior.

The authors declare no competing financial interests.

Correspondence should be addressed to Dr. Adriano B. L. Tort, Brain Institute, Federal University of Rio Grande do Norte, Rua Nascimento de Castro, 2155, Natal, RN 59056-450, Brazil. E-mail: tort@neuro.ufrn.br.

DOI:10.1523/JNEUROSCI.3452-15.2016

Copyright $\odot 2016$ the authors $\quad 0270-6474 / 16 / 365338-15 \$ 15.00 / 0$
(Sirota et al., 2003; Colgin et al., 2009; Schomburg et al., 2014). Much of the input to the hippocampus is sensorial, reaching the dentate gyrus (DG) and area CA1 through connections from the entorhinal cortex (Steward and Scoville, 1976; Burwell et al., 1995). Interestingly, activity at the early stages of sensory processing can be rhythmic too. Rodents breathe at a wide range of rates, from basal respiration $(<3 \mathrm{~Hz})$ to active sniffing $(>5 \mathrm{~Hz})(\mathrm{Wa}-$ chowiak, 2011), rhythmically activating sensory neurons in the olfactory epithelium (Verhagen et al., 2007). This periodicity is relayed to the olfactory bulb (OB) and to the piriform cortex, both of which show strong synchronization with the respiratory cycle (Adrian, 1942; Fontanini et al., 2003). These olfactory structures indirectly project to the hippocampus after a relay in the entorhinal cortex (Beckstead, 1978; Wilson and Steward, 1978; Schwerdtfeger et al., 1990). But whether hippocampal oscillatory activity synchronizes to respiration is still debated (Lockmann and Belchior, 2014). 
Recent efforts have explored this possibility in anesthetized preparations. During deep anesthesia, the rat hippocampus displays a slow oscillation ( $\mathrm{SO} ;<1.5 \mathrm{~Hz}$ ) that follows "up-and-down" transitions in the neocortex (Wolansky et al., 2006; Sharma et al., 2010), similarly to what happens in slow-wave sleep (Steriade et al., 1993a, b). In ketamine-anesthetized rats, Fontanini et al. (2003) reported oscillations of similar frequency in the piriform cortex, which were locked to the respiratory cycle. However, Viczko et al. (2014) convincingly demonstrated that hippocampal and neocortical SO recorded from rats during either urethane or ketamine anesthesia, as well as during natural slow-wave sleep, were not coupled to respiration. On the other hand, in the mouse hippocampus, Yanovsky et al. (2014) have recently described a low-frequency oscillation entrained by nasal respiration under urethane anesthesia; this rhythm, dubbed "hippocampal respiration-induced rhythm" (HRR), was most prominent in the DG and could be distinguished from simultaneously occurring theta waves. Therefore, whether low-frequency oscillations in the rodent hippocampus couple or not to respiration is at issue.

The opposite conclusions reached by Yanovsky et al. (2014) and Viczko et al. (2014) could be due to intrinsic differences in the studied species (mice vs rats), or, perhaps more likely, due to the fact that the two studies investigated different brain states (Lockmann and Belchior, 2014). Under urethane anesthesia, brain activity spontaneously switches between "activated states" (studied in Yanovsky et al., 2014) characterized by theta oscillations, and "deactivated states" (studied in Viczko et al., 2014) characterized by large-amplitude SO (Wolansky et al., 2006).

Here we have concomitantly recorded respiration along with hippocampal, neocortical, and OB LFPs in rats anesthetized with urethane. During the course of anesthesia, time-resolved spectral analyses showed that hippocampal LFPs transitioned between activity states characterized by the emergence of different oscillations. By jointly analyzing multisite LFP recordings and respiratory cycles, we could distinguish 3 types of independent oscillatory activity: SO, HRR, and theta. Theta oscillations were faster $(>3 \mathrm{~Hz})$, whereas HRR and SO had peak frequency in the range of $0.5-1.5 \mathrm{~Hz}$. And although theta and SO were typically mutually exclusive, HRR could coexist with either rhythm. Our results therefore solve the apparent contradictions among previous studies (Fontanini et al., 2003; Viczko et al., 2014; Yanovsky et al., 2014) by demonstrating that the rat hippocampus can produce two types of oscillations at frequencies $<1.5 \mathrm{~Hz}$ : one that is entrained to the respiratory cycle (HRR) and another that phaselocks to neocortical up-and-down transitions (SO).

\section{Materials and Methods}

Ethics statement. All experimental procedures were approved by the Ethical Committee for Animal Experimentation of the Federal University of Rio Grande do Norte, protocol number 044/2014. The directives of the Ethical Committee for Animal Experimentation of the Federal University of Rio Grande do Norte are in compliance with the Brazilian federal law for animal experimentation.

Subjects. Experiments were performed in 18 male Wistar rats weighting 300-420 g provided by the animal facilities of the Brain Institute of the Federal University of Rio Grande do Norte. The animals were housed in groups of 4, and kept on a $12 \mathrm{~h}$ light/dark cycle with food and water available ad libitum.

Surgical procedures. Subjects were anesthetized with intraperitoneal injections of $500 \mathrm{mg} / \mathrm{ml}$ urethane solution (U2500 Sigma, dissolved in saline). The total dose of $1.5 \mathrm{~g} / \mathrm{kg}$ was administered by three injections separated by $20 \mathrm{~min}$. Rectal temperature was monitored and maintained at $37^{\circ} \mathrm{C}-38^{\circ} \mathrm{C}$ by a thermal pad. Anesthesia induction was certified by the absence of withdrawal reflex to hindpaw pinching. Supplemental urethane doses of $0.3 \mathrm{~g} / \mathrm{kg}$ were administered as needed. Before surgery, 0.5 $\mathrm{ml}$ of lidocaine chlorhydrate $2 \%$ was subcutaneously applied in the scalp for local anesthesia; an intramuscular injection of $0.1 \mathrm{ml}$ of dexamethasone $2.5 \mathrm{mg} / \mathrm{ml}$ was also applied to prevent brain swelling.

After anesthetic induction, animals were placed in a stereotaxic frame (RWD Life Science), and a scalp incision was made to expose the skull. A stainless-steel screw placed at the interparietal bone was used as reference. In 6 animals, single wire recording electrodes (described below) were placed at OB (AP: $8.5 \mathrm{~mm}, \mathrm{ML}:-1.0 \mathrm{~mm}$, DV: $-1.6 \mathrm{~mm}$ ), CA1 (AP: $-3.5 \mathrm{~mm}$, ML: $-2.0 \mathrm{~mm}, \mathrm{DV}:-2.5 \mathrm{~mm}$ ), and DG (AP: $-3.5 \mathrm{~mm}$, ML: $-2.0 \mathrm{~mm}, \mathrm{DV}:-3.5 \mathrm{~mm}$ ) (Paxinos and Watson, 2004). In 4 of these animals, we additionally recorded from the medial prefrontal cortex (AP: $3.0 \mathrm{~mm}, \mathrm{ML}:-0.5 \mathrm{~mm}, \mathrm{DV}:-3.0 \mathrm{~mm}$ ). Dental acrylic cement was used for the fixation of single wire electrodes. All recordings were performed in the right hemisphere.

In 12 animals ( 6 intact, 6 tracheotomized), we recorded from the OB with a single wire and inserted a multichannel silicon probe in the hippocampus at AP: $-3.5 \mathrm{~mm}$ and ML: $-2.0 \mathrm{~mm}$. The deepest probe contact was placed at $-4.1 \mathrm{~mm}$ DV. To determine the location of probe contacts, stimulation electrodes were implanted at the perforant path (PP) (AP: $-7.3 \mathrm{~mm}, \mathrm{ML}:-5.0 \mathrm{~mm}, \mathrm{DV}:-2.5 \mathrm{~mm}$ ) and at the lateral olfactory tract (LOT) (AP: $3.0 \mathrm{~mm}, \mathrm{ML}:-3.5 \mathrm{~mm}, \mathrm{DV}:-6.4 \mathrm{~mm}$ ), and fixed with dental acrylic. In Figures 9 and 10, we only analyzed data from intact animals $(n=6)$.

Tracheotomy. Tracheotomy was performed before placing the animal in the stereotaxic frame. This surgery consisted of exposing the trachea, followed by an incision between the sixth and seventh cartilaginous rings to separate the trachea in two segments (rostral and caudal). In the 3 animals used in air puffing experiments (see Fig. 13), the tracheostomy was irreversible and animals breathed exclusively through a Teflon tube inserted into the caudal trachea. In 3 other animals, a Teflon tube was also inserted into the rostral tracheal segment. In these animals, air flowed only through the caudal tracheal tube when the tubes were disconnected, thus bypassing the nasal cavity. When the rostral and caudal tubes were connected, air flowed through the nasal cavity. This preparation permitted reversible switching between nasal and tracheal respiration (see Fig. 12).

Respiration recording. An 18-gauge stainless-steel cannula was used to record nasal and tracheal air pressure. Nasal pressure was recorded from a cannula placed in the nasal cavity (accessed through the nasal bone). Tracheal pressure was recorded using a cannula inside the caudal tracheostomy tube. Cannulas were connected to an analog pressure sensor amplifier (Honeywell 24PCAFA6G), and the output signals were recorded through auxiliary channels of our recording system (RZ2, Tucker-Davis Technologies). Downward and upward deflections in air pressure recordings correspond to inspiratory and expiratory phases, respectively, of the respiratory cycle.

Air puffing into nasal cavity. In 3 tracheotomized animals, air puffs were applied into the nasal cavity through an additional 18-gauge cannula placed inside a hole drilled in the right nasal bone (rostral in relation to the pressure recording cannula). This cannula was connected to a cylinder of compressed air using a PTFA tube. Airflow through the tube was controlled with a solenoid valve (075P2NC12-01SQ, Bio-Chem Fluidics) opened by $100 \mathrm{~ms}$ duration TTL pulses generated by the recording system (RZ2, Tucker-Davis Technologies). For each recording, air pressure was set so that single puffs evoked intranasal pressure signals of $1-2 \times$ the amplitude of the normal respiratory cycle. After calibration, air puffs were delivered rhythmically at $1 \mathrm{~Hz}$.

LFP recordings. For recordings from multiple brain areas, we used single Teflon-coated stainless-steel wires with $127 \mu \mathrm{m}$ of bare diameter (7914 AM Systems). For simultaneous multisite recordings along the dorsoventral hippocampal axis, we used 32-channel silicon probes (Neuronexus, A1x32-5 mm-100-413). Recording sites were $100 \mu \mathrm{m}$ spaced and had $28 \mu \mathrm{m}$ of bare diameter. Signals were amplified and digitized at 24,414 Hz (PZ2 and RZ2, Tucker-Davis Technologies). No digital filters were applied during the recording. All recordings were referenced to the interparietal screw. LFPs were obtained by low-pass filtering $(<500 \mathrm{~Hz})$ and down-sampling to $1000 \mathrm{~Hz}$. 
A

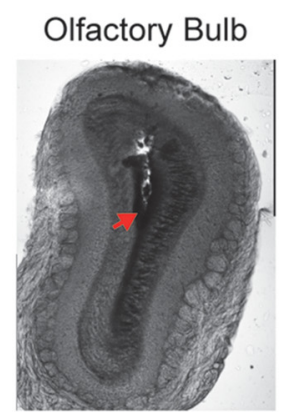

Single wire

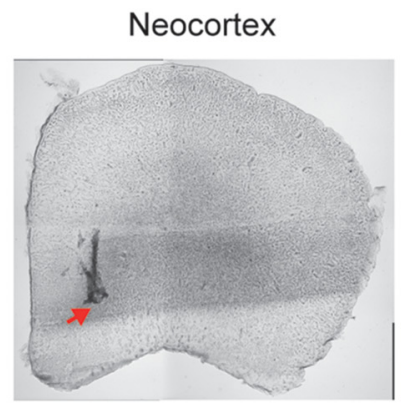

CA1 and DG

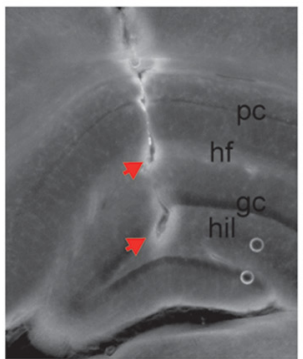

B Linear probe

\section{CA1-DG axis}

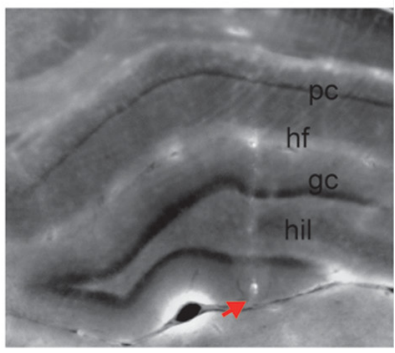

Figure 1. Histological positions of recording sites. $\boldsymbol{A}$, Example histology of single wires placed in $\mathrm{OB}$, neocortex, and hippocampus. $\boldsymbol{B}$, Example histology of a linear probe placed across the hippocampus. Red arrows indicate estimated positions of electrode tips. pc, Pyramidal cell layer; hf, hippocampal fissure; gc, granule cell layer; hil, hilus.

A

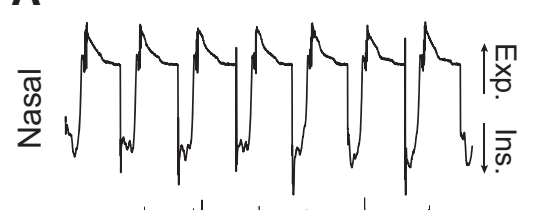

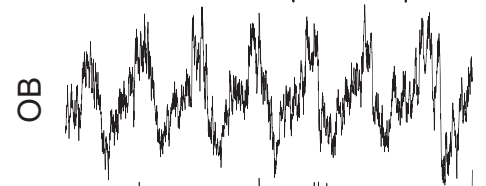
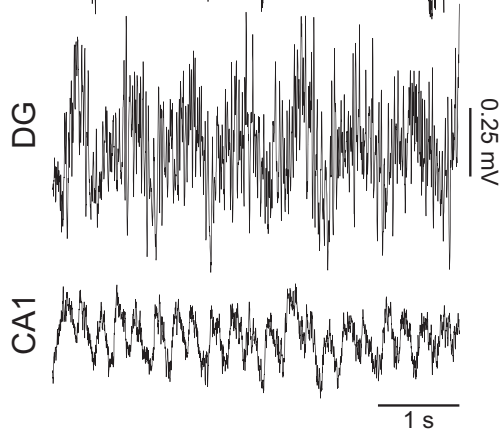

B

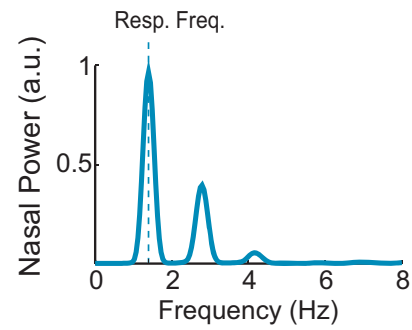

C
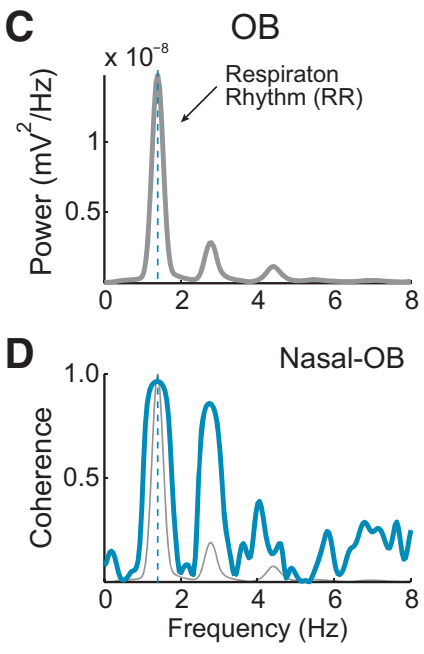

E

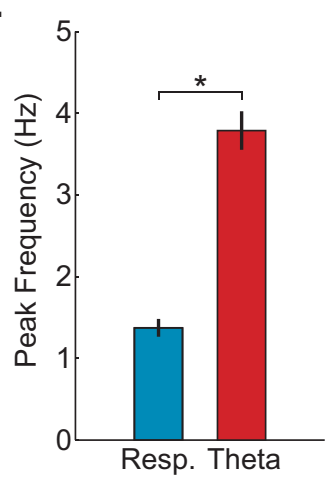

DG

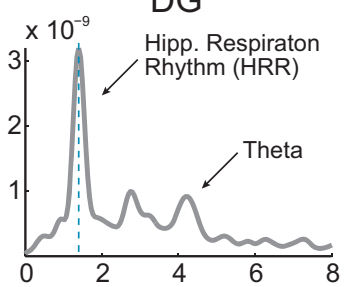

Nasal-DG

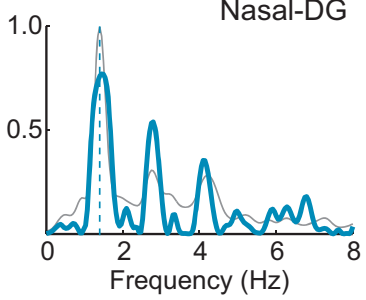

$\mathbf{F}$

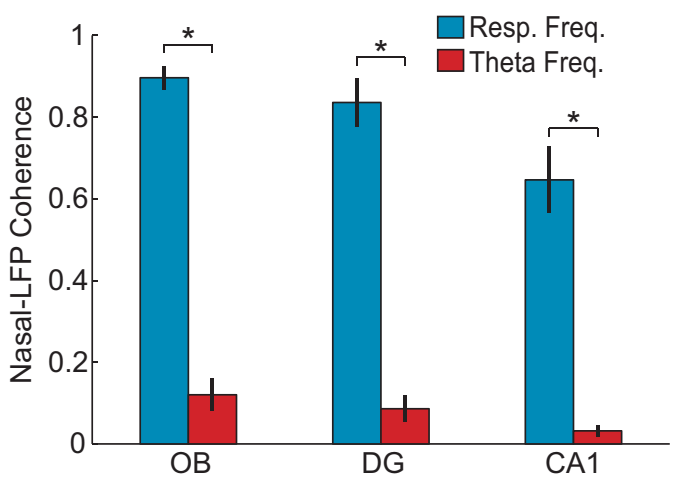

Figure 2. The rat hippocampus displays a low-frequency respiration-coupled oscillation (HRR), which coexists with faster theta oscillations during activated states. $\boldsymbol{A}$, Example raw traces of simultaneous recordings of air pressure in the nasal cavity (nasal) along with LFPs from the OB and hippocampus of a urethane-anesthetized rat (DG; CA1, region 1 of cornu ammonis). $\boldsymbol{B}$, Power spectrum of the nasal pressure signal, revealing a respiratory rate of $\sim 1.5 \mathrm{~Hz}$ (Resp. Freq., blue dashed line). $C, 0 B, D G$, and CA1 power spectra. There are prominent power peaks in $0 B$ and $D G$ at the respiratory frequency (blue dashed lines); $C A 1$ has a prominent power peak at the theta frequency range $(\sim 4.5 \mathrm{~Hz})$. $D$, Coherence spectra between nasal respiration and LFP signals (blue lines). Light gray lines reproduce the power spectra depicted in $C$. There are coherence peaks at the exact same frequency as respiration and its harmonics, along with lack of coherence at the peak frequency of CA1 theta. Results in $\boldsymbol{B}-\boldsymbol{D}$ were obtained from a 60 s representative data segment of activated state, which included the raw signals depicted in $\boldsymbol{A}$. $\boldsymbol{E}$, Mean respiratory and theta peak frequencies across animals ( $n=6$ rats). ${ }^{*} p<0.001$ (paired $t$ test). $\boldsymbol{F}$, Mean nasal-LFP coherence at respiratory and theta peak frequencies $\left(n=6\right.$ rats). ${ }^{*} p<0.001$ (paired $t$ tests). Error bars indicate SEM.

Electrical microstimulation. Bipolar stimulation electrodes consisted of a twisted pair of Teflon-coated stainless-steel wire (7914 AM-Systems) with $1 \mathrm{~mm}$ distance between electrode tips. Electrical stimuli were generated by a current isolator (ISO-Flex, A.M.P.I.), which was set to deliver $100 \mu$ s constant current pulses with various amplitudes. The stimulus amplitude was set as the minimum current to evoke a visible deflection in the raw signal (range: $100-250 \mu \mathrm{A}$ ). The stimuli were triggered by TTL pulses (RZ2, Tucker-Davis Technologies) at $0.05 \mathrm{~Hz}$. The electrical stimulation protocol consisted of a train of 10 stimuli first to the PP and then to the LOT. Evoked potentials were obtained by averaging $100 \mathrm{~ms}$ LFP segments locked to stimulus onset. The hilus of the DG was identified as the channel with the highest positive component of the PP-evoked potential (Canning and Leung, 1997). Probe recordings from different animals were aligned using the DG hilus as reference. The hippocampal 

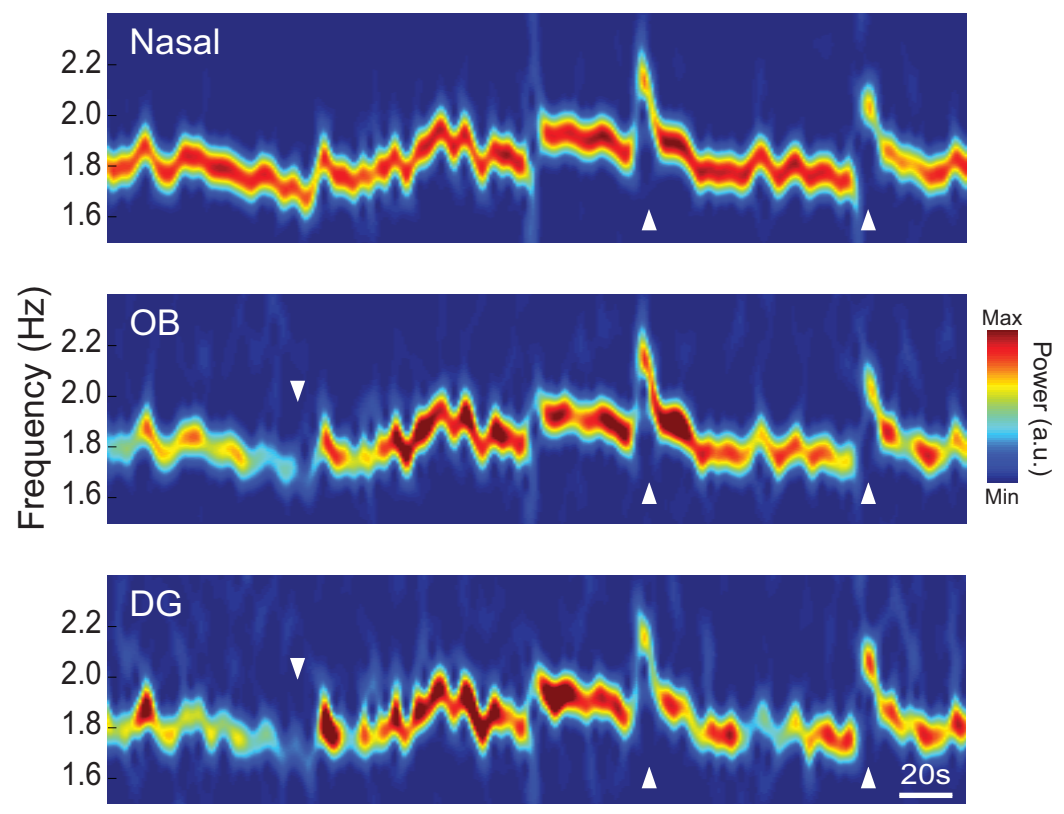

Figure 3. The peak frequency of respiration-coupled LFP rhythms in DG and OB follows variations in breathing rate. Example of time-frequency power analysis of a nasal pressure recording (top) and of LFPs simultaneously recorded from OB (middle) and DG (bottom) in a representative animal. Upward arrowheads indicate moments of sharp increase in respiratory frequency. There are similar changes in peak frequency for all signals. Downward arrowhead indicates a period in which OB and DG failed to follow the nasal signal.

fissure was identified as the channel with the maximum theta power (Brankačk et al., 1993).

Perfusion and histology. After the recordings, the animals were transcardially perfused with $0.9 \%$ sodium chloride followed by $4 \%$ PFA. Brains were removed and stored in 4\% PFA; $120 \mu \mathrm{m}$ coronal sections were made in a vibrating tissue slicer (EMS) and mounted on glass slides. Electrode tracks were visualized and photographed in a microscope equipped with epifluorescence (Zeiss). Figure 1 shows example histological sections of recorded sites.

Data analysis: spectral analysis. Recorded data were analyzed offline using built-in and custom-written MATLAB codes (The MathWorks). Power spectra were calculated by means of Welch's periodogram (built-in MAT$\mathrm{LAB}$ pwelch function). Coherence spectra of signal pairs were computed using magnitude-squared coherence (built-in MATLAB mscohere function). Both power and coherence spectra calculations were performed in $60 \mathrm{~s}$ data segments using $4 \mathrm{~s}$ Hamming windows with $90 \%$ overlap. Time-frequency power decomposition was calculated by means of the built-in MATLAB spectrogram function. In Figures 3, 12, and 13, spectrograms were calculated using $7 \mathrm{~s}$ sliding Hamming windows with $140 \mathrm{~ms}$ time steps; in Figures 8 and 11, $10 \mathrm{~s}$ sliding Hamming windows with $1 \mathrm{~s}$ time steps were used.

LFP staging. LFP activity was divided in two categories according to the predominant frequency band in the power spectrum (Wolansky et al., 2006): (1) activated LFP states, which were characterized by theta oscillations (3-7 Hz); and (2) deactivated LFP states, characterized by largeamplitude SO $(<1.5 \mathrm{~Hz})$. Urethane-anesthetized rats normally show spontaneous cyclical transitions from deactivated to activated states (Clement et al., 2008; Sharma et al., 2010). In some recordings, the activated state was induced by continuously pinching the animal tail (see Fig. 8 ). For the summary statistics shown in Figures $5 B$ and 7, we used nonoverlapping epochs within deactivated states from the 6 animals implanted with single wires and the 6 intact animals implanted with silicon probes. We used $30 \mathrm{~s}$ epochs in Figure $5 B$ and $60 \mathrm{~s}$ epochs in Figure 7 . For each animal, we analyzed $\sim 1$ h of concatenated deactivated state periods. In Figure 7, HRR was assumed to coexist with SO if DG LFP coherence with the nasal pressure signal was $>0.4$.

Laminar profile. The laminar profile is a plot of the average amplitude of an oscillation as a function of the recording depth. For estimating the laminar profile of HRR, we selected for each animal a $100 \mathrm{~s}$ period with high HRR power. LFPs were filtered between 0.3 and $2 \mathrm{~Hz}$ using the eegfilt function from the EEGLAB toolbox (Delorme and Makeig, 2004). For each channel, the respiration-triggered HRR average was obtained as follows: we first localized the timestamps associated with the peak values of the simultaneously recorded nasal pressure signal (built-in MATLAB findpeaks function); we then extracted nonoverlapping $3 \mathrm{~s}$ filtered LFP epochs centered on these timestamps; finally, the average HRR trace was obtained by computing the mean over all 3 s epochs.

For the SO laminar profile, a $100 \mathrm{~s}$ period with high SO power was selected for each animal, and LFPs were low-pass filtered $<2 \mathrm{~Hz}$. For each channel, the SO-triggered LFP average was obtained as the mean over nonoverlapping $3 \mathrm{~s}$ filtered LFP epochs centered on the SO peak times, which were identified from the more superficial probe channel. Theta laminar profile followed exactly the same method, except that we selected periods of high theta power and filtered the LFP signals between 3 and $7 \mathrm{~Hz}$.

Current source density (CSD) was estimated as the second spatial derivative of voltage recorded from linear probe contacts, as previously described (Brankačk et al., 1993).

Statistics. Group data are expressed as mean \pm SEM. Statistical differences were assessed by paired $t$ test (built-in MATLAB ttest function).

\section{Results}

A respiration-coupled LFP rhythm in the hippocampus of urethane-anesthetized rats distinct from theta oscillations During urethane anesthesia, hippocampal field potentials have been previously shown to display spontaneous transitions between "activated states" (dominated by $\sim 4 \mathrm{~Hz}$ theta) and "deactivated states" (dominated by $\sim 1 \mathrm{~Hz}$ SO coupled to neocortical up-and-down transitions) (Wolansky et al., 2006; Clement et al., 2008; Sharma et al., 2010). Accordingly, in our recordings (18 rats across all experiments), theta oscillations regularly emerged in the course of anesthesia alternating with periods of large amplitude $\mathrm{SO}$ activity.

Figure $2 A$ shows raw recordings during a representative period in which theta was prominent in the hippocampus. During these activated states, we observed that the hippocampus could concurrently display an additional rhythm at a lower frequency than theta. Different from theta, which was dominant in CA1, such low-frequency rhythm appeared more notably in DG (Fig. $2 A, C)$. Noteworthy, whereas LFPs from CA1 and DG were markedly different (compare the raw traces in Fig. $2 A$ ), DG and OB LFPs were somewhat similar (Fig. $2 A, C$ ). Compared with the nasal pressure recording, the cycles of the low-frequency rhythm in $\mathrm{OB}$ and DG showed a 1:1 correspondence with the breathing cycles (Fig. 2A). Spectral analysis of OB and DG LFPs evidenced a prominent power peak exactly at the respiratory frequency (Fig. $2 B, C)$; CA1 also exhibited a power peak at the respiratory frequency, but much smaller than the power peak at theta. In addition, coherence between nasal respiration and LFPs was high at the respiratory frequency and its harmonics, but not at theta frequency (Fig. 2D). Therefore, the low-frequency oscillation in DG matched the frequency of nasal respiration and was phasecoupled to the breathing cycles. These results extend to rats the recent findings of Yanovsky et al. (2014), who reported a "hippocampal respiration-induced rhythm" (HRR) in urethaneanesthetized mice. Henceforth, we will use a similar terminology 
A

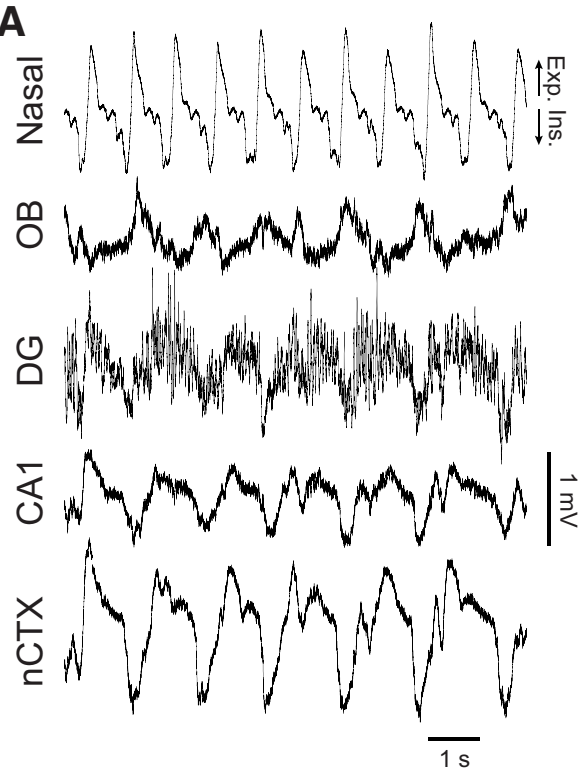

C

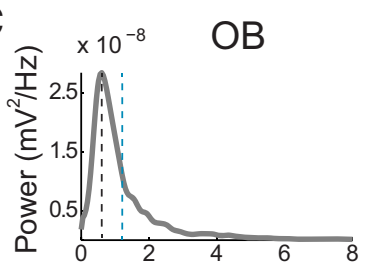

D
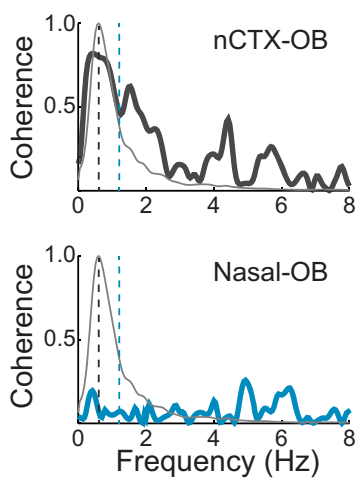
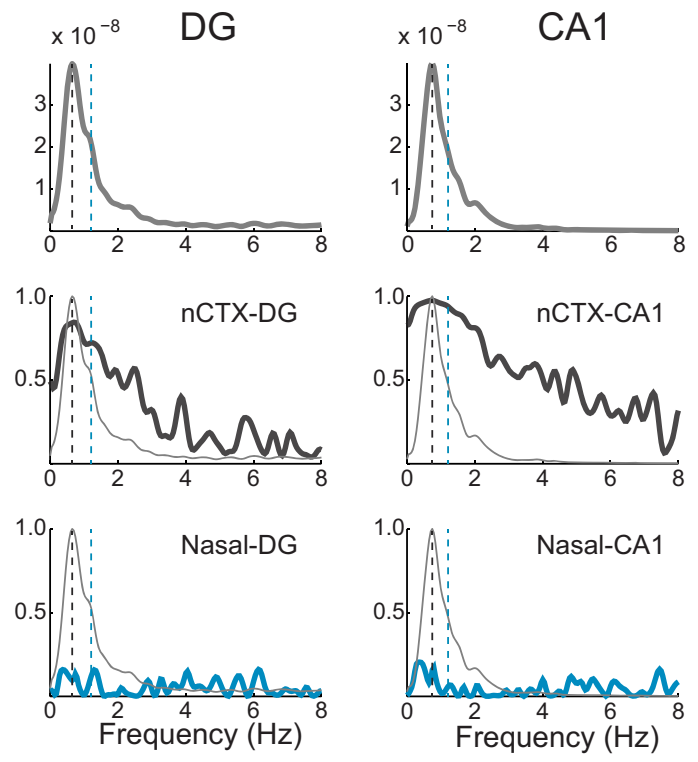

B

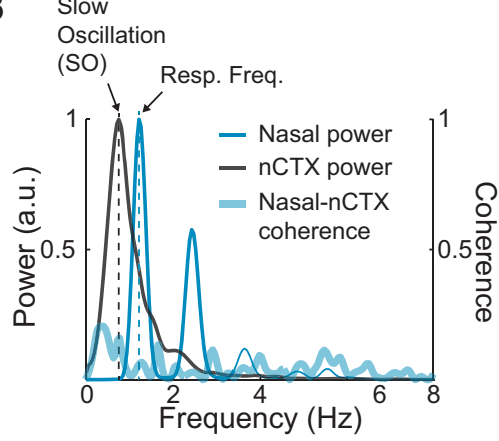

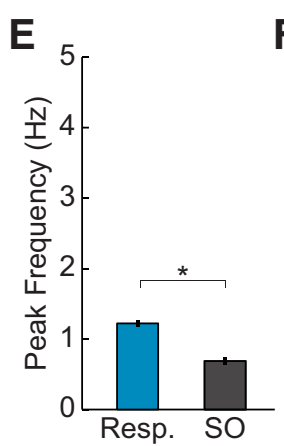
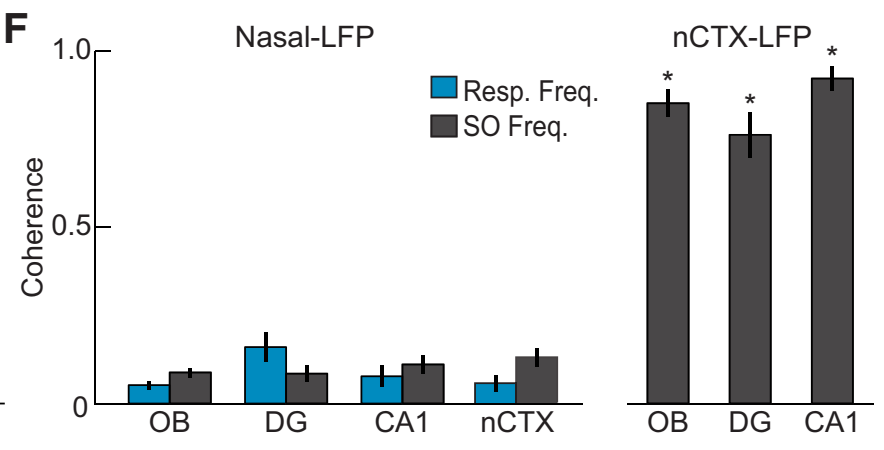

Figure 4. The rat hippocampus displays a low-frequency oscillation, which is entrained to neocortical SO, but not to respiration, during deactivated states. $\boldsymbol{A}$, Representative nasal pressure and LFP signals recorded during large-amplitude SO in the neocortex (nCTX). B, Power spectra of the nasal signal (blue) and nCTX LFP (dark gray) (left $y$-axis). Blue and black dashed lines indicate the respiratory and SO peak frequencies, respectively. Thick blue line indicates the coherence spectrum between nCTX and the nasal signal (right $y$-axis). C, Power spectra of OB and hippocampal LFPs, which show a power peak $\sim 1 \mathrm{~Hz}$ coinciding with the nCTX SO peak frequency. $\boldsymbol{D}$, Top, nCTX-LFP coherence spectra, revealing coherence peaks at the SO frequency. Bottom, Nasal-LFP coherence spectra. There is low coherence at all frequencies. Light gray lines reproduce the power spectra in $\boldsymbol{C}$. Results in $\boldsymbol{B}-\boldsymbol{D}$ were obtained from a 60 s representative data segment of deactivated state, which included the raw signals depicted in $\boldsymbol{A}$. $\boldsymbol{E}$, Mean respiratory and SO peak frequencies ( $n=4$ rats). ${ }^{*} p<0.01$ (paired $t$ test). $\boldsymbol{F}$, Left, Mean nasal-LFP coherence at respiratory and SO peak frequencies. Right, Mean nCTX-LFP coherence at SO peak frequency ( $n=4$ rats). ${ }^{*} p<0.001$, compared with nasal-LFP at SO frequency (paired $t$ tests). Error bars indicate SEM.

and refer to the respiration-coupled LFP rhythm in the rat hippocampus as HRR, and in OB as RR. Of note, the HRR we describe here is slower than in Yanovsky et al. (2014) because rats breathe slower than mice (see Discussion).

To compute group data, we selected, for each animal, activated LFP periods when the two rhythms (theta and HRR) coexisted for at least $60 \mathrm{~s}$. During such periods, the respiratory peak frequency, and by extension also RR and HRR peak frequency, was consistently lower than theta peak frequency (Fig. 2E; $1.37 \pm$ 0.11 vs $3.79 \pm 0.24 \mathrm{~Hz}, n=6$ rats, $t_{(5)}=15.66, p<0.001$, paired $t$ test). Hence, whenever the hippocampus displayed both rhythms, HRR appeared in the power spectrum as an isolated peak at a lower frequency than theta (Fig. 2C).

Figure $2 F$ shows the mean coherence between LFPs and the nasal respiration signal captured by the pressure sensor in the same analyzed epochs as above. As expected, OB and DG were highly coherent with the pressure signal at the respiratory frequency across animals. Interestingly, even though CA1 had low HRR activity, it was similarly highly coherent with respiration (Fig. $2 F$ ). In contrast, LFP coherence with the nasal respiration signal at theta frequency was significantly lower in all recorded regions $\left(n=6\right.$ rats, OB: $t_{(5)}=11.91$, DG: $t_{(5)}=9.94$, CA1: $t_{(5)}=7.47$, all $p$ values $<0.001$, paired $t$ tests).

When looking at longer epochs associated with variable breathing rate, time-resolved spectral analysis showed that the peak frequency of RR and HRR closely followed changes in instantaneous breathing rate (for an example, see Fig. 3). Interestingly, the OB LFP could occasionally "fail" to follow nasal respiration, and these periods coincided with disappearance of HRR in DG (Fig. 3, downward arrowhead).

In all, these results demonstrate the existence of a respirationcoupled rhythm in the rat hippocampus, which is clearly distinct from theta oscillations.

\section{Independent respiration- and neocortex-coupled low-} frequency rhythms in the hippocampus

Activated states alternated with a state in which theta disappeared and a slower hippocampal rhythm of large amplitude emerged (Fig. 4A; compare with Fig. 2A). Simultaneously recorded LFP in neocortex displayed the characteristic SO (Fig. $4 A, B$ ), which has been shown to reflect periodic membrane potential changes from depolarization to hyperpolarization (up-and-down transitions) 
A
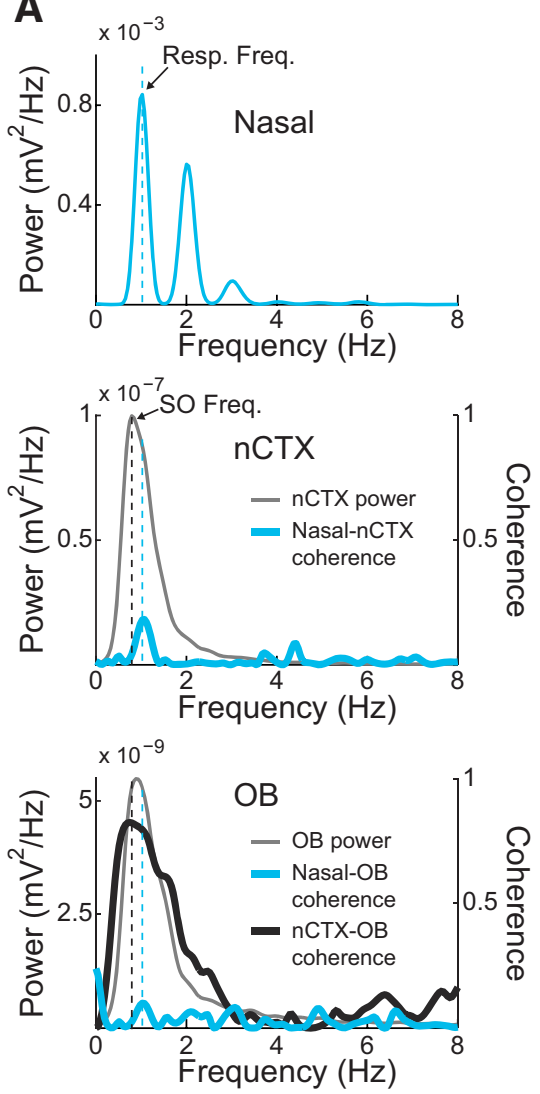

B
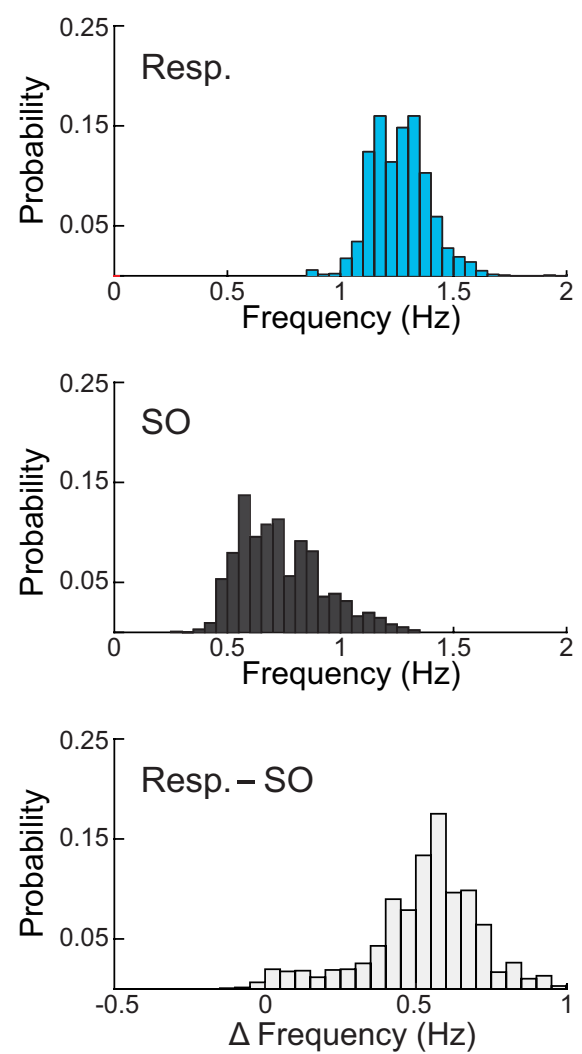

Figure 5. High LFP power at the respiratory peak frequency is not sufficient for high coherence between respiration and LFP. $\boldsymbol{A}$, Example power and coherence spectra during a deactivated state period. Bottom, Despite high OB power at the respiratory peak frequency (blue dashed line), nasal-OB coherence is low (thick blue trace). On the other hand, $\mathrm{nCTX}-\mathrm{OB}$ coherence is high (thick black trace), revealing therefore that the $O B$ power peak corresponds to $S 0$ and not RR. $B$, Distribution of respiration (top) and SO (middle) peak frequencies, as well as of their frequency differences (bottom), assessed in 30-s-long epochs ( $n=1370$ epochs across 12 rats).

during slow-wave sleep and deep anesthesia (Steriade et al., 1993a, b). The slow LFP rhythm in CA1 and DG was associated with a power peak at the same frequency as the neocortical SO (Fig. 4C) and, moreover, was highly coherent with it (Fig. 4D). During these periods, a similar large-amplitude rhythm could also be seen in $\mathrm{OB}$, though with inversed polarity (Fig. 4A,C). Thus, both $\mathrm{OB}$ and hippocampus may exhibit a neocortexcoupled slow rhythm during deactivated brain states. We will refer to this rhythm also as SO to stress that it entrains with concurrent neocortical SO and differs from the respiration-coupled LFP rhythm. This nomenclature is also in line with previous research that has extensively characterized this oscillation in the hippocampus (Wolansky et al., 2006). The high coherence between hippocampal and neocortical SO observed here is similar to that previously reported (Wolansky et al., 2006; Sharma et al., 2010).

The entrainment of all recorded regions by SO during deactivated states markedly contrasts with what we observed in the activated states, when local hippocampal circuits expressed distinct oscillatory patterns (theta in CA1 along with HRR in DG). It is currently debated whether SO reflects a global brain entrainment to rhythmic nasal respiration (Fontanini et al., 2003; Fontanini and Bower, 2006; Viczko et al., 2014). Indeed, SO frequency is quite close to the respiratory frequency of urethaneanesthetized rats. However, by comparing SO-entrained LFP traces with nasal respiration, it was clear that these signals were not synchronous (Fig. 4A). Power spectra of neocortical LFP and nasal pressure signals evidenced that rhythmic respiration and $\mathrm{SO}$ had near but distinct peak frequencies (Fig. 4B). Across animals, SO frequency was significantly lower than the respiratory frequency (Fig. $4 E ; 0.69 \pm 0.04$ vs $1.22 \pm 0.05 \mathrm{~Hz}, n=4$ rats, $t_{(3)}=17.82, p<0.001$, paired $t$ test; see also Fig. $5 B$ ). Further supporting that $\mathrm{SO}$ is independent of respiration, LFP Coherence with nasal respiration was low at all frequencies, whereas, as expected, $\mathrm{OB}$ and hippocampal LFP coherence with the neocortex was high at the SO frequency (Fig. $4 D, F)$. Coherence with neocortex was also high at the respiratory frequency (Fig. 4D); this, however, does not reflect a respiration-coupled rhythm (given no coherence with respiration) but is rather due to the large width of the SO power peak, which includes the respiratory frequency. To further illustrate this point, Figure $5 \mathrm{~A}$ shows an example of SO coherence between $\mathrm{OB}$ and neocortex at a similar frequency as respiration. $\mathrm{SO}$ and respiration frequencies differed by $<0.3 \mathrm{~Hz}$ in $11.5 \%$ of $30 \mathrm{~s}$ epochs within deactivated states (Fig. 5B).

Interestingly, during the course of anesthesia, animals also exhibited periods within deactivated states in which HRR coexisted with SO (Fig. 6). HRR could be observed in raw LFPs as sharp peaks superimposed at random phases of the $\mathrm{SO}$ cycle (Fig. 6A, more easily noticeable in $\mathrm{OB}$ and DG traces), which were instead synchronized to the respiratory cycle (Fig. $6 A$, blue arrowheads and vertical line). Spectral analysis confirmed the presence of robust rhythmic activity at the exact same frequency as respiration (Fig. $6 B, C$ ) and coherent with it (Fig. 6D). Noteworthy, respiration-coupled oscillations could also be observed in the neocortex (Fig. 6A,B); indeed, during these periods, all recorded regions (OB, hippocampus, and neocortex) exhibited two power peaks at frequencies $<1.5 \mathrm{~Hz}$ : one corresponding to $\mathrm{SO}$ and the other to (H)RR.

Whenever SO and HRR coexisted, the higher-frequency peak in the power spectrum coincided with the respiration peak frequency. Group results confirmed that HRR was significantly faster than SO during these epochs (Fig. $6 E ; 1.25 \pm 0.10$ vs $0.64 \pm$ $0.05 \mathrm{~Hz}, n=4$ rats, $t_{(3)}=11.50, p<0.01$, paired $t$ test). Importantly, high LFP coherence with the nasal respiration signal occurred only at the respiratory peak frequency but not at the SO frequency (Fig. $6 D, F ; n=4$ rats, OB: $t_{(3)}=16.99$, DG: $t_{(3)}=$ 26.49, CA1: $t_{(3)}=14.99$, nCTX: $t_{(3)}=20.61$, all $p$ values $<0.001$, paired $t$ tests). Nevertheless, coherence with neocortex was consistently high at SO frequency (Fig. $6 D, F ; n=4$ rats, OB: $t_{(3)}=$ 43.35, DG: $t_{(3)}=16.45$, CA1: $t_{(3)}=61.68$, all $p$ values $<0.001$, paired $t$ tests compared with nasal-LFP coherence at SO frequency). These findings further reinforce that $\mathrm{SO}$ is not entrained by the nasal respiratory rhythm (as reported by Viczko et al., 2014) while also showing that a low-frequency respirationcoupled rhythm may exist in the hippocampus during deactivated states (as reported in olfactory cortex by Fontanini et al., 

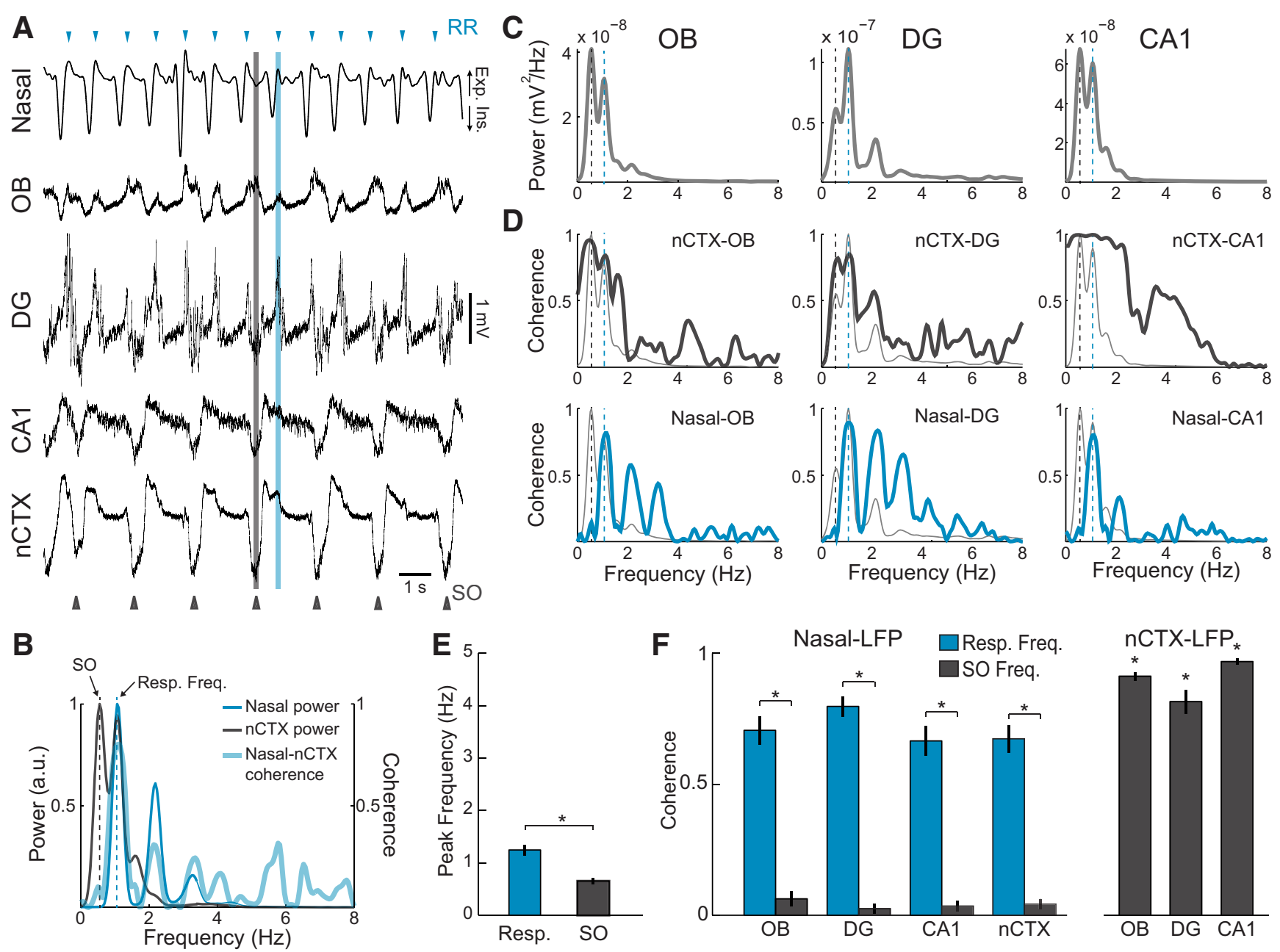

Figure 6. Respiration- and neocortex-coupled hippocampal rhythms can simultaneously occur. $\boldsymbol{A}$, Representative nasal pressure and LFP signals recorded during SO activity in the neocortex (nCTX). Blue arrowheads indicate the peaks of the respiration-coupled rhythm (RR) in OB. Black arrowheads indicate the troughs of neocortical SO. Black and blue vertical lines highlight SO- and respiration-coupled LFP components, respectively. B, Power spectra of the nasal signal (blue) and nCTX LFP (dark gray) (left $y$-axis). Thick blue line indicates nasal-nCTX coherence (right $y$-axis). $\boldsymbol{C}$, Power spectra of LFPs, revealing a power peak (black dashed line) coinciding with the nCTX SO peak frequency and another power peak coinciding with the respiratory peak frequency (blue dashed line). D, nCTX-LFP (top) and nasal-LFP (bottom) coherence spectra. Light gray lines reproduce the power spectra depicted in $\boldsymbol{C}$. There is high coherence with both neocortical SO and nasal respiration at the corresponding frequencies. Results in $\boldsymbol{B}-\boldsymbol{D}$ were obtained from a 60 s representative data segment of a deactivated state, which included the raw signals depicted in $\boldsymbol{A}$. $\boldsymbol{E}$, Mean respiratory and SO peak frequencies ( $n=4$ rats). ${ }^{*} p<0.01$ (paired $t$ test). $F$, Left, Mean nasal-LFP coherence at respiratory and SO peak frequencies. Right, Mean nCTX-LFP coherence at SO peak frequency ( $n=$ 4 rats). ${ }^{*} p<0.001$, compared with nasal-LFP at $S 0$ frequency (paired $t$ tests). Error bars indicate SEM.

2003). Across 12 animals ( 6 rats recorded with single wires and 6 with silicon probes; used below), hippocampal SO coexisted with HRR in $48.3 \pm 9.2 \%$ of deactivated periods (Fig. 7 ).

In all, the results confirm the recent findings of Viczko et al. (2014) of lack of respiratory coupling with hippocampal SO. Therefore, just by looking at different anesthesia periods within the same experimental setup, we could corroborate the apparently contradictory conclusions of Yanovsky et al. (2014) (Fig. 2) and Viczko et al. (2014) (Fig. 4), and also extend these findings by showing that HRR can co-occur not only with theta but also with SO (Fig. 6). Figure 8 shows an example of transition between activated and deactivated states in which the three oscillatory patterns (SO, HRR, and theta) can be observed. Of note, we found no coupling between HRR phase and theta amplitude during activated states or between SO phase and HRR amplitude during deactivated states (data not shown).

\section{HRR has different laminar profile from SO and theta}

The multiple hippocampal layers along the CA1-DG axis receive specific synaptic inputs that are associated with distinct patterns of rhythmic LFP activity (Colgin et al., 2009; Scheffer-Teixeira et al., 2012; Schomburg et al., 2014). In the experiments above, we simultaneously recorded from CA1 and DG using single wires, and, consistent with Yanovsky et al. (2014), found HRR to be larger in DG than CA1 recordings. We next set out to better characterize the anatomical profile of HRR. To that end, we used a 32-channel linear probe (100 $\mu \mathrm{m}$ separation between channels), which permitted simultaneous LFP recordings from all layers of the dorsal hippocampus.

The entorhinal cortex is likely to mediate the entry of olfactory information to the hippocampus, as it receives direct inputs from the OB and piriform cortex (Canto et al., 2008). The entorhinal cortex in turn sends projections to CA1 via the temporoammonic pathway and to DG via the PP (Steward and Scoville, 1976). It has been largely documented that electrical stimulation of the PP leads to highest evoked potential response at the DG hilus (Buzsáki et al., 1986; Canning and Leung, 1997). Figure 9A (left) shows a representative laminar profile of evoked responses to PP stimulation reproducing this effect. Interestingly, electrical stimulation of the LOT, the main axonal bundle leaving OB, evoked 
A

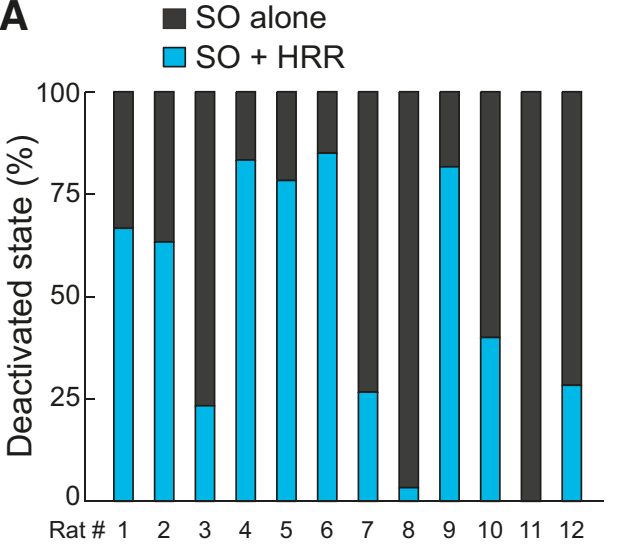

B

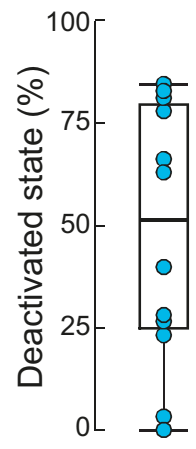

Figure 7. Percentage of $\mathrm{SO}$ and HRR coexistence during deactivated states. $\boldsymbol{A}$, Percentage of 60 s epochs within deactivated states with $\mathrm{SO}$ alone or with $\mathrm{SO}$ coexisting with HRR for each animal. HRR was defined to occur when nasal-LFP coherence was $>0.4$ at the respiratory frequency. $\boldsymbol{B}$, Boxplot distribution of the percentage of $\mathrm{SO}$ and $\mathrm{HRR}$ coexistence within deactivated states across animals ( $n=12$ ).

maximal response also at the depth of the hilus (Fig. $9 A$, middle), although at a longer latency (Fig. $9 B$; PP latency: $6.5 \pm 0.7 \mathrm{~ms}$; LOT: $24.0 \pm 1.6 \mathrm{~ms}, n=6$ rats, $t_{(5)}=11.17, p<0.001$, paired $t$ test). This suggests that activity propagating from the $\mathrm{OB}$ to the hippocampus arrives at the DG after a relay in the entorhinal cortex, consistent with previous reports (Wilson and Steward, 1978; de Curtis et al., 1991; Vanderwolf, 1992).

We then proceeded to compute the laminar distribution of HRR activity across the dorsoventral axis of the hippocampus (Fig. 9A, right; see Materials and Methods). Consistent with Yanovsky et al. (2014), we found that HRR had maximal amplitude at the hilus of the DG, and, indeed, the laminar profile of HRR looked identical to that of the evoked responses to either PP or LOT stimulation (Fig. 9C). In contrast, the laminar profile of $\mathrm{SO}$ and theta oscillations revealed a different picture, in which theta was maximal at the hippocampal fissure (Buzsáki et al., 1986; Brankačk et al., 1993), while SO exhibited rather modest changes in amplitude across the hippocampus (Fig. 10) (Wolansky et al., 2006); of note, during probe insertion, we could observe that SO phase-reverses within the parietal cortex as described by Wolansky et al. (2006) and Sharma et al. (2010). Thus, despite the similarities in frequency range, these results show that HRR and SO have distinct laminar profiles, and hence are generated by different network mechanisms.

Finally, we went on to analyze CSD signals (Fig. 11). Raw CSD signals also exhibited HRR activity, which was highest at the DG hilus (Fig. $11 A-C$ ), consistent with HRR voltage laminar profile (Fig. 9). Moreover, as with LFP recordings, during activated brain states raw CSD signals could simultaneously exhibit HRR and theta (Fig. 11D) while during deactivated states HRR could coexist with SO (Fig. 11E).

\section{Rhythmic nasal airflow is necessary and sufficient to drive coherent low-frequency oscillations in OB and DG}

Respiratory rhythmicity in the rodent brain originates in two distinct circuits (Kleinfeld et al., 2014). Specific clusters of neurons in the brainstem generate periodic efferent signals that drive inspiration/expiration cycles (Feldman and Del Negro, 2006). In turn, the resulting rhythmic airflow through the nasal cavity activates olfactory sensory neurons (Grosmaitre et al., 2007; Verhagen et al., 2007) and imposes a reafferent periodicity in down-

stream olfactory areas (Adrian, 1942). We next investigated which of the two mechanisms (brainstem vs reafferent signal) drives HRR. By means of reversible tracheostomy, we first addressed the effect of switching off nasal airflow on HRR.

Before opening the tracheostomy, rats breathed rhythmically through the nose and LFPs displayed prominent RR and HRR (Fig. $12 A, B$ ). In addition, LFP coherence with the nasal pressure signal, as well as OB-DG coherence, was high at the respiratory frequency (Fig. 12D). The rate of nasal respiration was equivalent in tracheotomized and intact rats (compare Figs. $12 C$ and $2 E$ ). After tracheostomy opening, nasal airflow immediately vanished as rats started to breathe through the trachea (Fig. 12A-C). During tracheal respiration, the brainstem continued to generate the rhythmical drive to control breathing; measures of air pressure inside the trachea confirmed that breathing rate was equal in nasal and tracheal respiration conditions (Fig. 12C; nasal: $1.13 \pm$ $0.19 \mathrm{~Hz}$, tracheal: $1.14 \pm 0.29 \mathrm{~Hz}, n=3$ rats, $t_{(2)}=0.16, p=0.88$, paired $t$ test). Following the removal of nasal airflow, RR and HRR disappeared (Fig. 12A,B), together with LFP coherence with respiration (Fig. 12D,E; assessed from tracheal pressure; $n=3$ rats, OB: $t_{(2)}=4.37, p<0.05$, DG: $t_{(2)}=12.32, p<0.01$, paired $t$ tests). In addition, during tracheal breathing OB-DG coherence drastically decreased (Fig. $12 D, E ; n=3$ rats, $t_{(2)}=$ $8.89, p<0.05$, paired $t$ test). These results show that nasal airflow is necessary for RR and HRR.

We next assessed whether artificial nasal airflow suffices to reinstate coherent LFP oscillations by applying air puffs at $1 \mathrm{~Hz}$ into the nasal cavity of tracheotomized rats. While rhythmic nasal airflow and LFP respiration-coupled rhythms were initially absent in these animals, the delivery of $1 \mathrm{~Hz}$ air puffs generated rhythmic LFP activity in OB and DG (Fig. 13A,B). Timefrequency analysis revealed high power at $1 \mathrm{~Hz}$ in both nasal pressure and LFP signals during air puffs. LFP oscillations were highly coherent with the $1 \mathrm{~Hz}$ nasal pressure rhythm, and, similarly, OB-DG coherence also peaked at the air puff frequency (Fig. 13C; notice that tracheal respiration was slightly faster than $1 \mathrm{~Hz}$ ). Across animals, LFP coherence with the nasal pressure signal was much higher during air puffs compared with periods of tracheal respiration alone (Fig. $13 D ; n=3$ rats, OB: $t_{(2)}=5.71$, $p<0.05$, DG: $t_{(2)}=6.39, p<0.05$, paired $t$ tests). In stark contrast, LFP coherence with the tracheal pressure signal was low during either tracheal respiration alone or tracheal respiration along with air puffs (Fig. 13C,D).

Together, these results indicate that the reafferent signal originating in the nasal cavity, and not the efferent brainstem signal, drives RR and HRR.

\section{Discussion}

By performing a system-wide approach of simultaneously recording respiratory activity and LFPs from multiple brain regions, here we have shown that the rat hippocampus exhibits two types of low-frequency $(<1.5 \mathrm{~Hz})$ network oscillations during urethane anesthesia: SO and HRR. Our results further show that HRR may coexist with faster theta oscillations that characterize activated LFP states (Fig. 2), and also with SO activity during deactivated states (Fig. 6). The three rhythms differ from each other by several features, such as exact peak frequency, coherence with respiration, laminar profile across the CA1-DG axis, and sensitivity to tracheotomy.

Our work was motivated by 2 recent studies with apparently opposing conclusions: one that showed evidence against (Viczko et al., 2014) and one that showed evidence for (Yanovsky et al., 2014) the coupling of low-frequency hippocampal oscillations to 

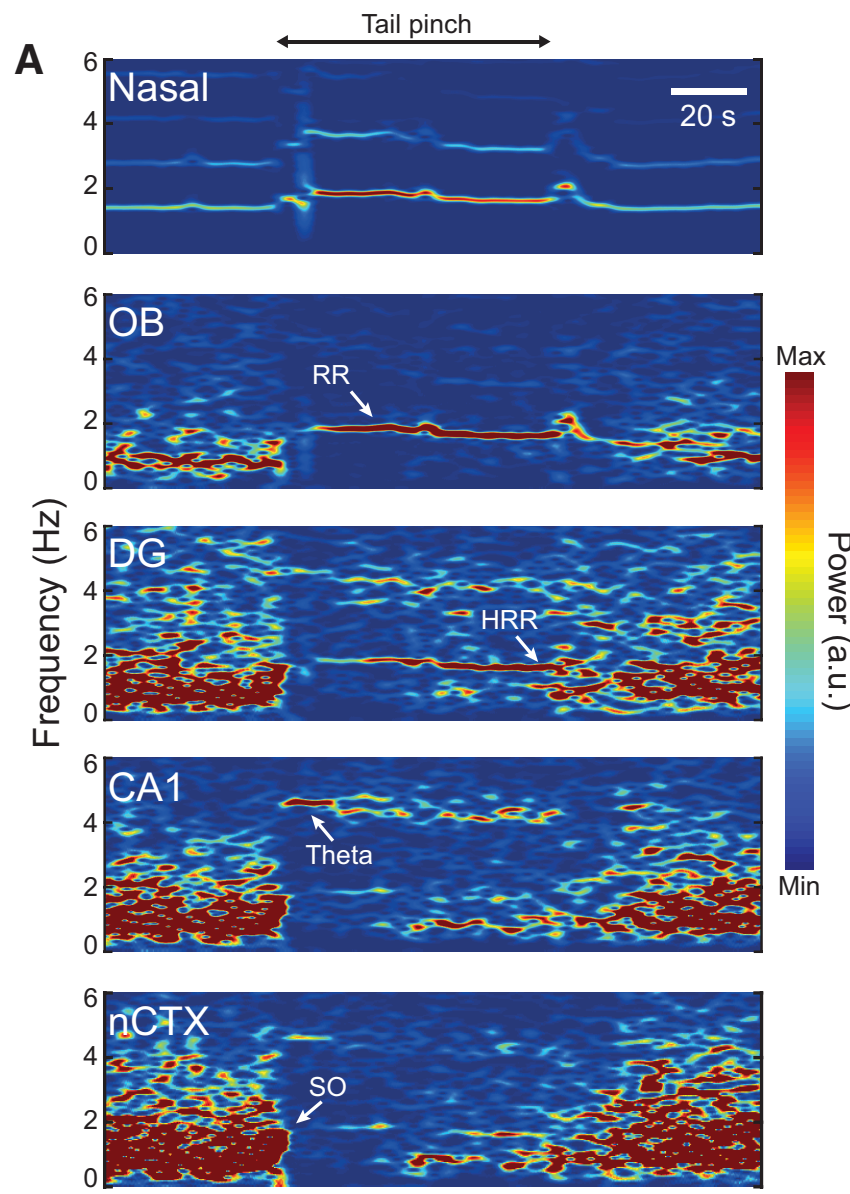

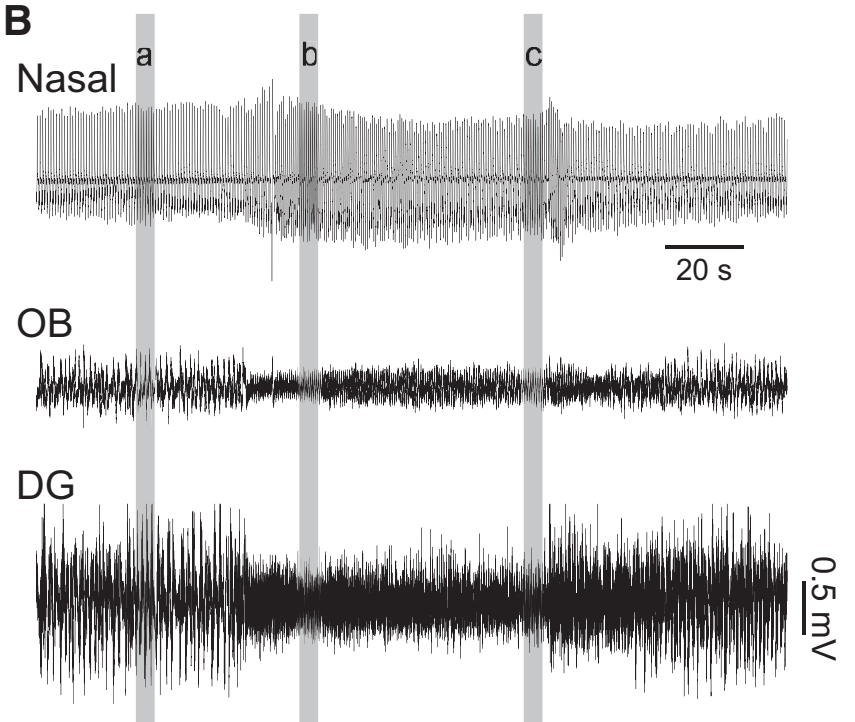

CA1

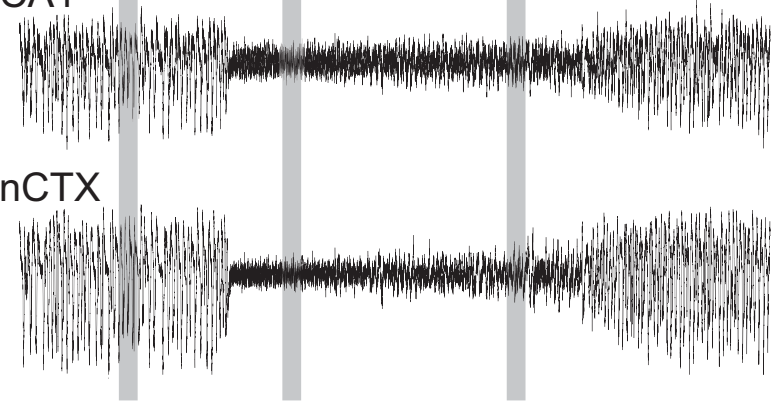

C

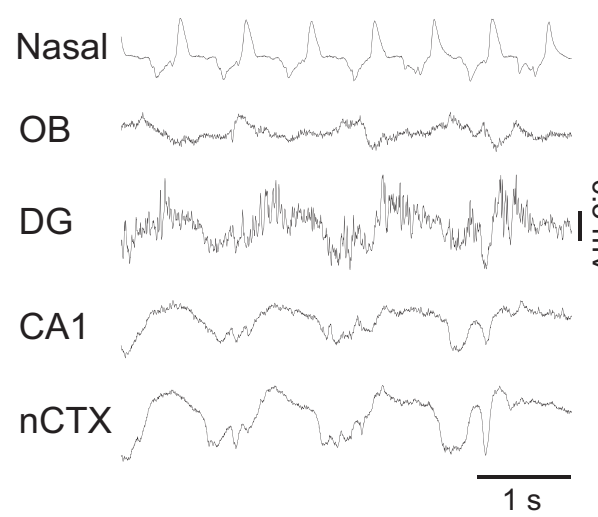

b. Theta

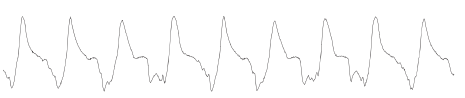

c. Theta to SO transition

Figure 8. Multiple oscillatory patterns appear in the hippocampus during transitions from deactivated to activated states. $\boldsymbol{A}$, Time-frequency power analysis of nasal pressure and LFP signals. Switching from deactivated to activated LFP states was induced by tail pinch (period between the black arrowheads). $\boldsymbol{B}$, Traces show the corresponding raw signals. $\boldsymbol{C}$, Zoomed-in view of the same signals during the three highlighted periods in $\boldsymbol{B}$. Before tail pinch $(\mathbf{C a})$, LFPs are dominated by large-amplitude SO activity. During tail pinch ( $\boldsymbol{C} b)$, RR becomes prominent in $\mathrm{OB}$, whereas theta and HRR dominate LFP activity in CA1 and DG, respectively. SO reemerges after several seconds of continued pinching (CC).

the respiratory cycle. Because both studies were well delineated and reached solid, though contradictory, results, we reasoned that their discrepancy should be due to methodological differences. While the main results of the two studies were obtained during anesthesia (similar protocol as used here), it is worth noting that: (1) they used different species (rats vs mice); (2) their major claims refer to different hippocampal subregions (CA1 vs DG); and (3) different LFP states were considered (deactivated vs activated).
The study of Viczko et al. (2014) constitutes a follow-up of previous research performed by the Dickson laboratory, which has been characterizing SO during sleep and anesthesia in the rat neocortex and hippocampus (Wolansky et al., 2006; Clement et al., 2008; Schall et al., 2008; Sharma et al., 2010; Pagliardini et al., 2012). Among other findings, this laboratory has been showing striking similarities between brain activity patterns during urethane anesthesia and natural sleep. Indeed, these authors promoted the use of the terms deactivated (for slow-wave sleep- 
A

$\downarrow$ PP stimulation

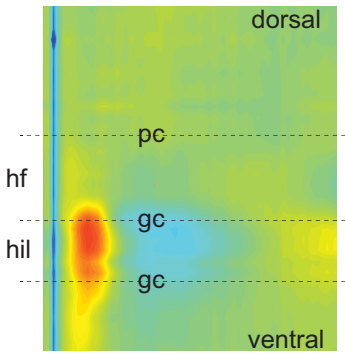

$\overline{30 \mathrm{~ms}}$
\ LOT stimulation

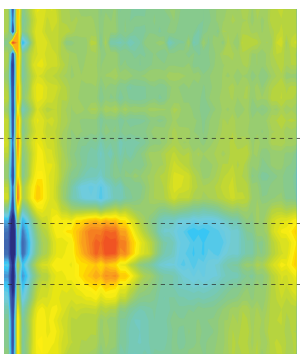

$\overline{30 \mathrm{~ms}}$
Respiration-triggered HRR

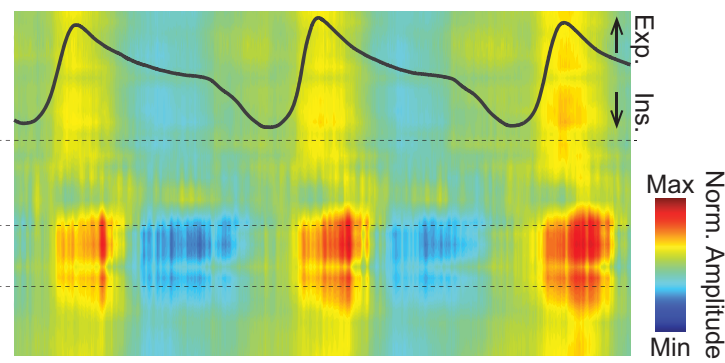

$500 \mathrm{~ms}$
B

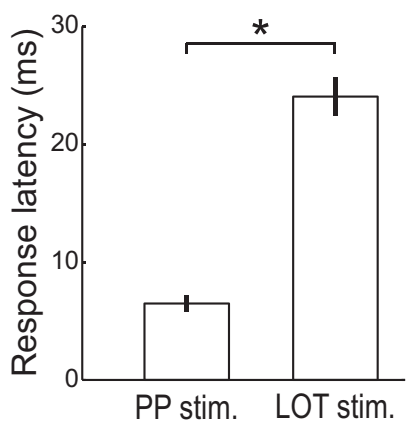

C

$$
\text { PP stim. }
$$

LOT stim.

HRR

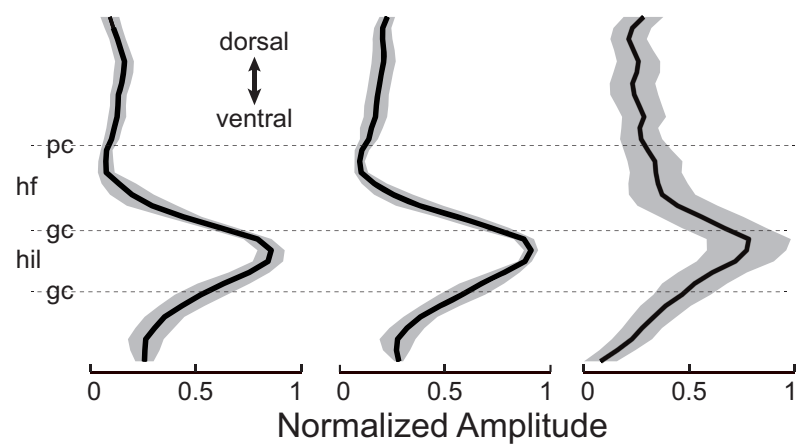

Figure 9. HRR has maximum amplitude in the DG hilus. A, Evoked potentials in the hippocampal dorsoventral axis to electrical stimulation of PP (left) and LOT (middle) in a representative animal. PP- and LOT-evoked potentials have maximum amplitude in the DG hilus. Arrows indicate stimulus artifact. Laminar profile of respiration-triggered average HRR activity reveals that HRR is also maximal in the hilus (right). $\boldsymbol{B}$, Mean ( \pm SEM) evoked potential latency to PP and LOT stimulation (hilus recordings, $n=6$ rats). ${ }^{*} p<0.001$ (paired $t$ test). $\boldsymbol{C}$, Mean amplitude of evoked potentials and HRR along the hippocampal dorsoventral axis $(n=6$ rats). Gray shades represent \pm SEM. Amplitudes were normalized within animals to achieve values between 0 (minimal) and 1 (maximal). pc, Pyramidal cell layer; hf, hippocampal fissure; gc, granule cell layer; hil, hilus.

A

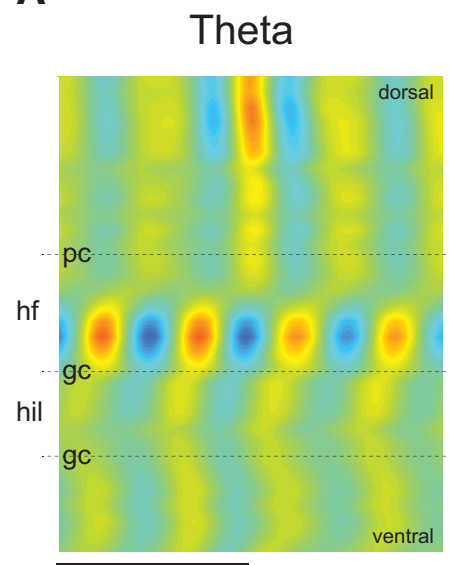

$500 \mathrm{~ms}$
Slow Oscillation

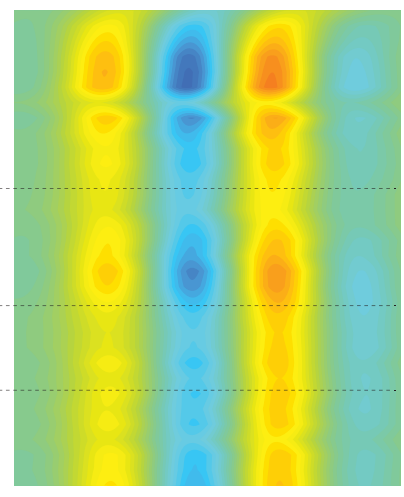

$500 \mathrm{~ms}$
B

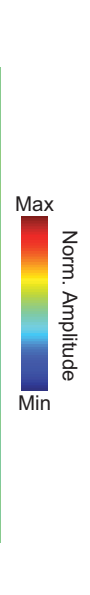

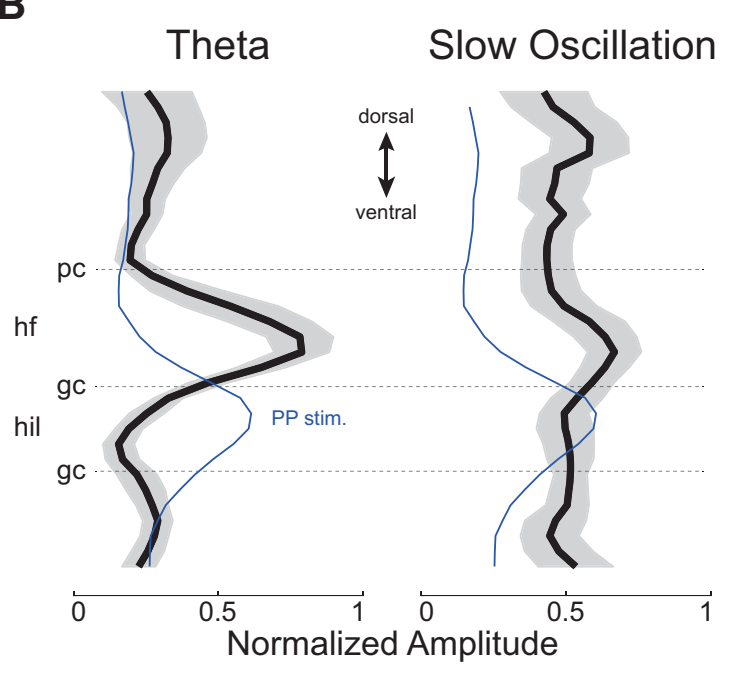

Figure 10. Theta and $S 0$ have maximum amplitude in the hippocampal fissure. $A$, Amplitude of theta and $S 0$ along the hippocampal dorsoventral axis. Data from activated (theta) and deactivated (SO) LFP periods of a representative animal. $\boldsymbol{B}$, Mean ( \pm SEM) theta and SO amplitude along the hippocampal dorsoventral axis $(n=6$ rats). Thin blue line indicates mean evoked potential to PP stimulation for reference. pc, Pyramidal cell layer; hf, hippocampal fissure; gc, granule cell layer; hil, hilus.

like/SO activity) and activated (for REM sleep-like/theta activity) brain states used in the present study. In support to their previous findings, Viczko et al. (2014) demonstrated that hippocampal SO indeed phase-locks to neocortical up-and-down transitions during sleep and deep anesthesia. Most importantly, they conclusively showed that hippocampal SO was not coupled to respiration. This result is relevant (actually the title of their work) because previous findings in the piriform cortex of rats by Fon- 
A
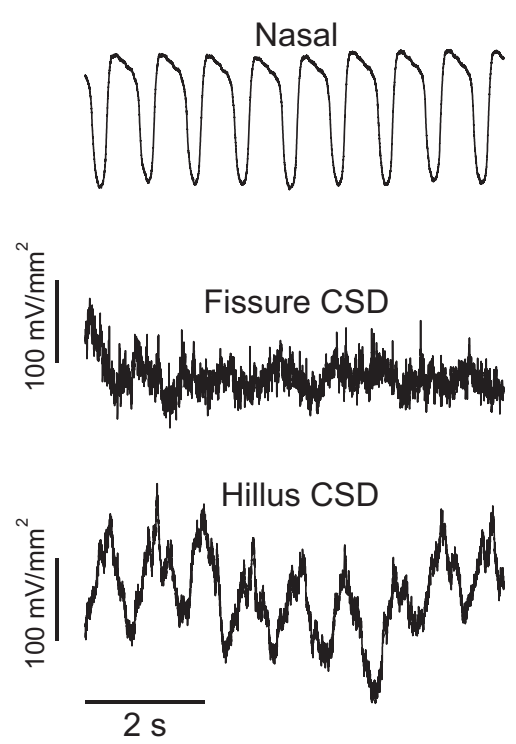

B

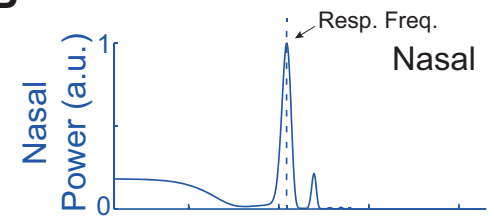

C

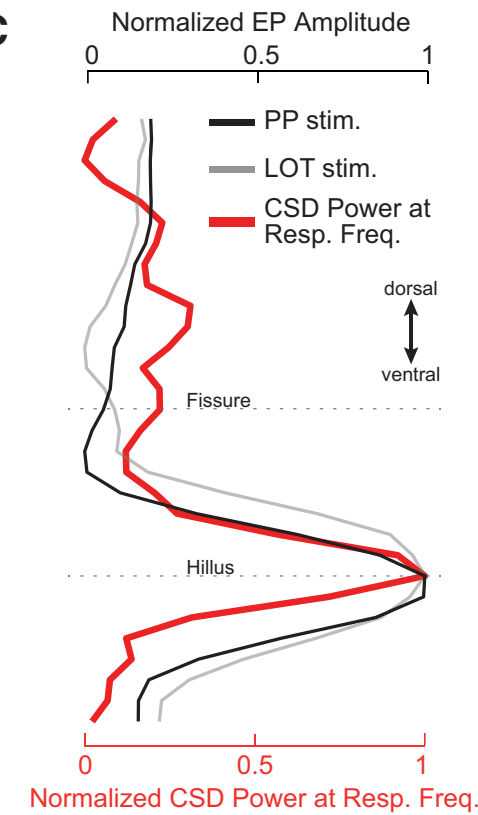

D
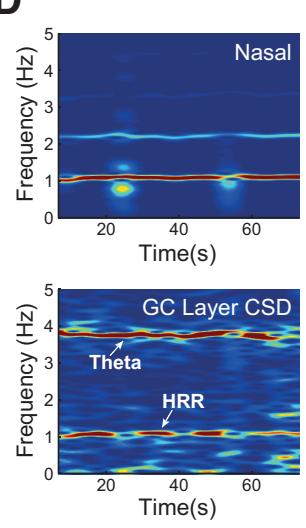
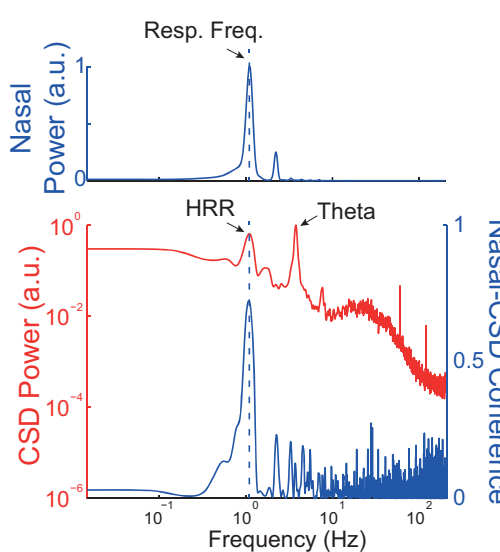

E
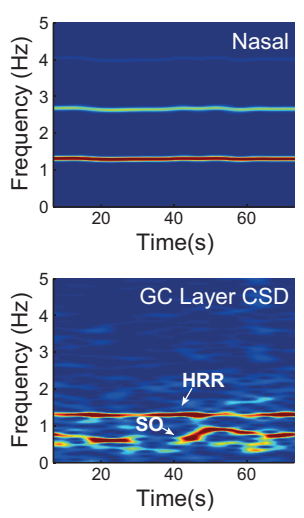

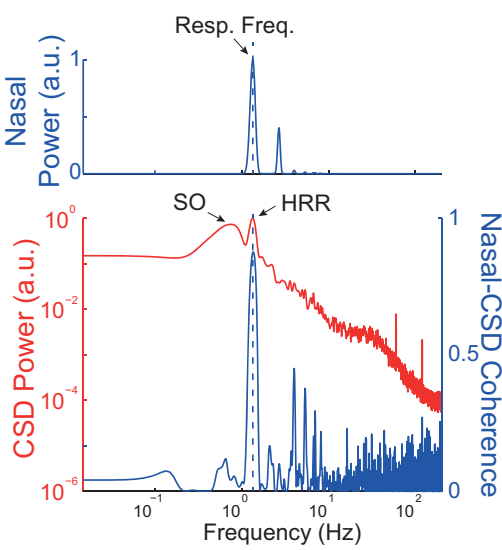

Figure 11. Raw CSD signals in DG exhibit prominent HRR. $\boldsymbol{A}$, Representative nasal pressure signal (top) and raw CSD traces from hippocampal fissure (middle) and DG hilus (bottom). There is prominent HRR in hilus CSD. B, CSD power and nasal-CSD coherence for the same signals as in $\boldsymbol{A}$ (analyzed time: 80 s). $\boldsymbol{C}$, Normalized CSD power at respiratory frequency (red) along the hippocampal dorsoventral axis. Black and gray lines indicate the amplitude of PP- and LOT-evoked potentials, respectively. $\boldsymbol{D}$, Left, Time-frequency power analysis of nasal pressure (top) and of granule cell layer CSD (bottom) during an activated state. There is coexistence of theta and HRR. Right, Power and coherence spectra for the same data, showing high coherence at the respiratory frequency. $E$, Same as $\boldsymbol{D}$, but for recordings obtained during a deactivated state. In this case, HRR coexists with $S 0$.

tanini and Bower had suggested a close link between respiration and low-frequency oscillations seen during ketamine anesthesia (Fontanini et al., 2003; Fontanini and Bower, 2005) and slowwave sleep (Fontanini and Bower, 2006). That is, Fontanini and Bower essentially hypothesized that up-and-down transitions would reflect coupling to respiratory inputs (Fontanini and Bower, 2006). Our results confirm the Viczko et al. (2014) conclusion that hippocampal SO is not coupled to respiration, but to neocortical SO. However, we also extended their findings by showing that SO can actually coexist with HRR. We suspect that previous studies linking low-frequency LFP oscillations to respiration (Fontanini et al., 2003; Fontanini and Bower, 2005) have actually detected a respiration-coupled rhythm but have not recognized a different LFP oscillation from SO due to the similarity in peak frequency. Interestingly, Viczko et al. (2014) also found that respiration- and neocortex-coupled low-frequency rhythms could coexist in the piriform cortex during the deactivated state; however, as their hippocampal recordings did not display HRR, but only SO, they concluded that "respiratory-related oscillatory neural activities are likely limited to primary olfactory structures during slow-wave forebrain states." We believe that Viczko et al. (2014) did not observe HRR because they restricted their recordings to CA1, where HRR is not as prominent as in DG (Figs. 9, 11).

The work of Yanovsky et al. (2014) demonstrated a lowfrequency hippocampal oscillation coupled to respiration during activated periods of urethane anesthesia in mice. Our work extends Yanovsky et al. (2014) by showing that HRR also exists in the rat hippocampus and that it can also emerge during deactivated LFP states. In further agreement with them, we found HRR to be more pronounced in DG than in CA1. Because mice breathe considerably faster than rats during anesthesia $(\sim 3.5 \mathrm{~Hz}$ vs $\sim 1.3$ $\mathrm{Hz}$, compare our Fig. $2 E$ with their Fig. 2D), Yanovsky et al. (2014) mainly coped with differentiating HRR from concomitant theta waves, which they succeeded to do. The HRR we describe here is slower than in mice and could be easily differentiated from theta. However, as respiratory rate in rats is closer to SO than 
A
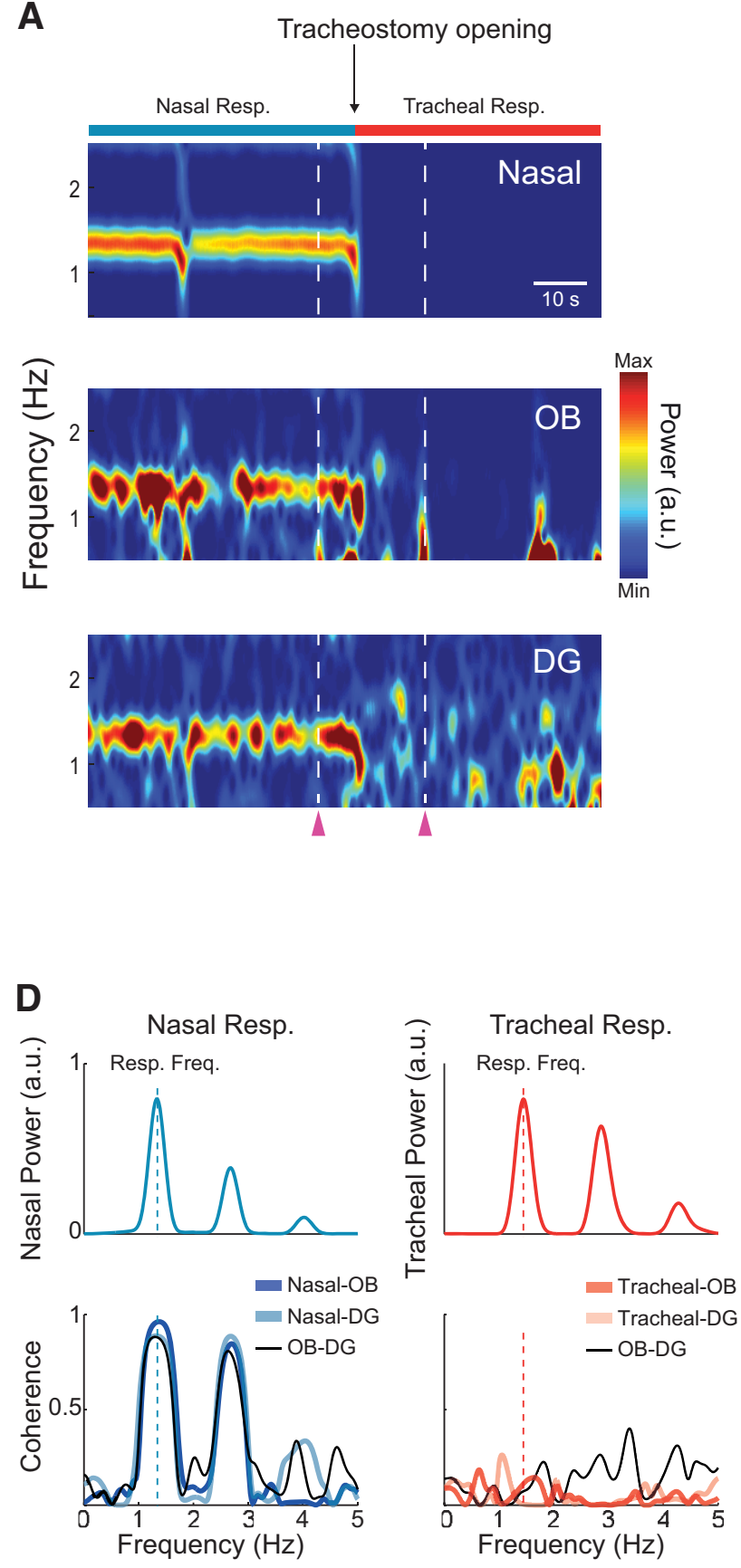

B
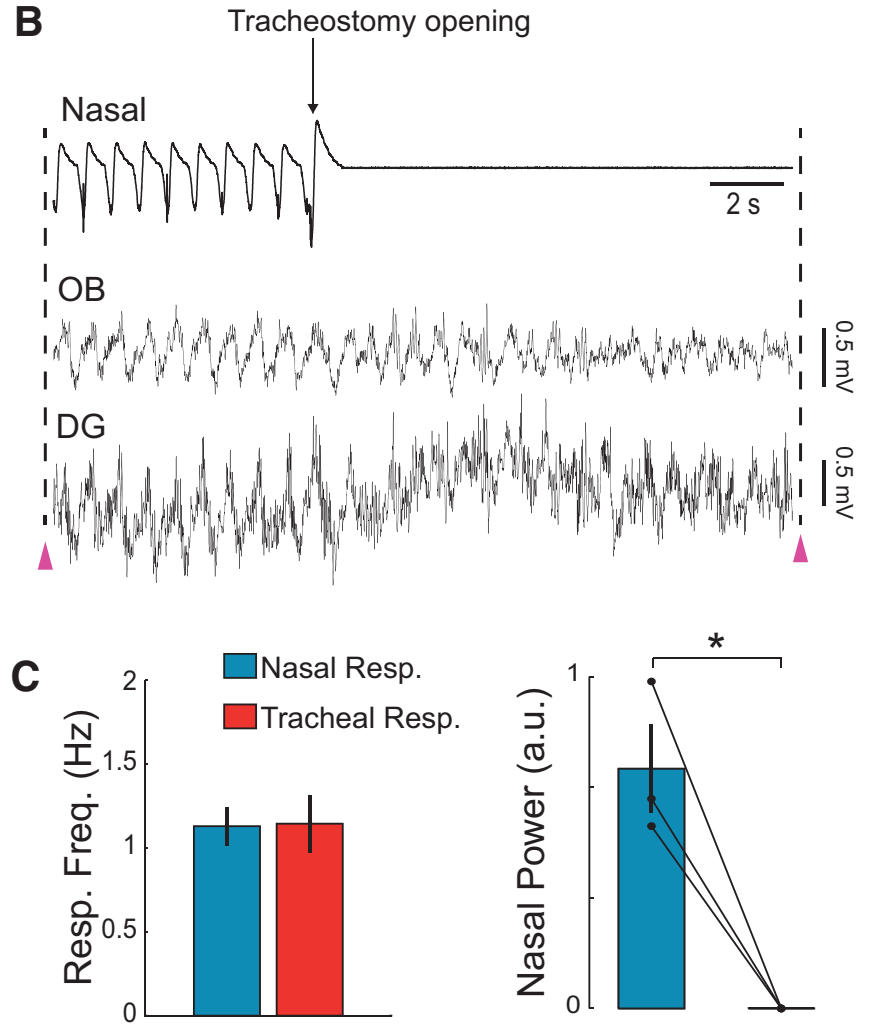

E

Nasal Resp.

Tracheal Resp.

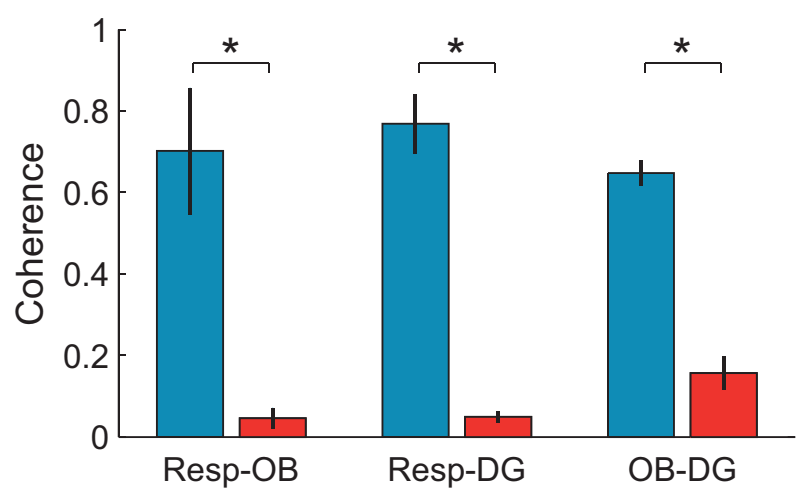

Figure 12. Interruption of nasal airflow by tracheotomy abolishes HRR. A, Time-frequency power analysis of nasal pressure and LFP signals recorded from a urethane-anesthetized rat breathing through reversible tracheostomy. Before the opening of the tracheostomy (Nasal Resp.), the LFPs exhibit prominent respiration-coupled rhythms (RR and HRR). After the opening of the tracheostomy (Tracheal Resp., onset indicated by the black arrow), the rhythmic airflow through the nasal cavity as well as RR and HRR vanish immediately. During tracheal respiration, air pressure was also recorded from the tracheal cannula to track respiratory frequency (data not shown; but see Fig. 13). $\boldsymbol{B}$, Raw signals for the period indicated by the purple arrowheads in $\boldsymbol{A}$. C, Left, Respiratory peak frequency during nasal (blue) and tracheal respiration (red). Right, Nasal pressure power at the respiratory peak frequency during nasal and tracheal respiration $\left(n=3\right.$ rats). ${ }^{*} p<0.05$ (paired $t$ test). D, Left, Power spectrum of nasal pressure during nasal respiration (top), and nasal-LFP and OB-DG coherence spectra for the same period (bottom). There is high coherence at the respiratory frequency. Right, Power spectrum of tracheal pressure during tracheal respiration (top), along with tracheal-LFP and OB-DG coherence (bottom). There is lack of coherence among signals during tracheal respiration. $E$, Mean $\left( \pm\right.$ SEM) respiration-LFP and OB-DG coherence at the respiratory frequency during nasal (blue bars) and tracheal respiration (red bars) $\left(n=3\right.$ rats). ${ }^{*} p<0.05$ (paired $t$ tests).

theta, in the present work we have focused on distinguishing HRR from SO.

We believe our work reconciles the dispute as to whether SO couples or not to respiration in rats (Fontanini et al., 2003; Fontanini and Bower, 2006; Viczko et al., 2014) by showing that there are actually two distinct activity patterns with overlapping frequency ranges. One of them (here called (H)RR) indeed couples to the respiratory cycle, whereas the other pattern is not entrained to respiration but to neocortical SO. To be in line with previous nomenclature, we refer to this latter rhythm also as SO (Steriade et al., 1993b; Wolansky et al., 2006). Indeed, much of the controversy mentioned above may actually be due to a rather semantic issue. Several laboratories worldwide still classify brain oscillations solely based on their frequency range, which the current 
A

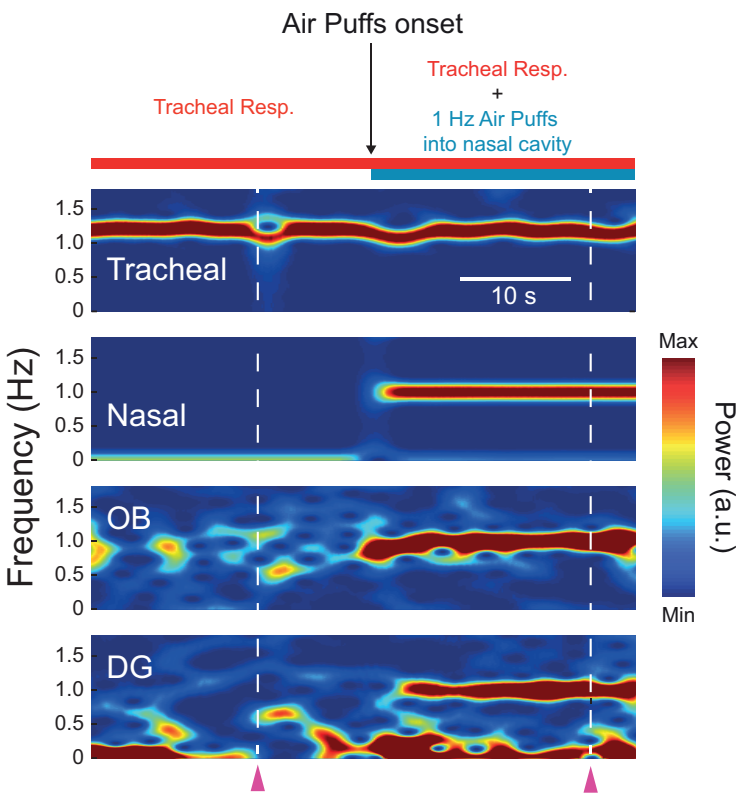

C
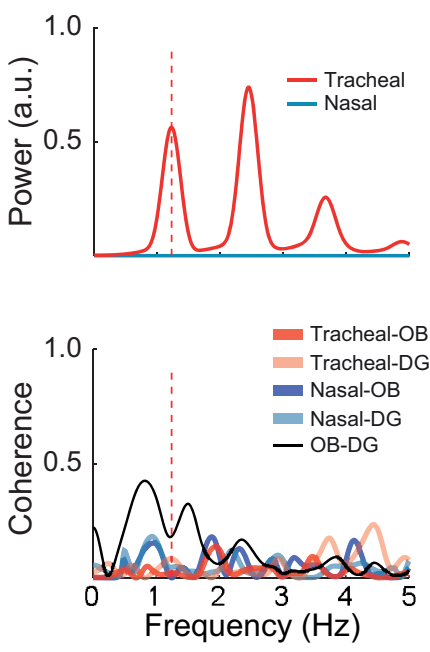

Tracheal Resp. + Air Puff
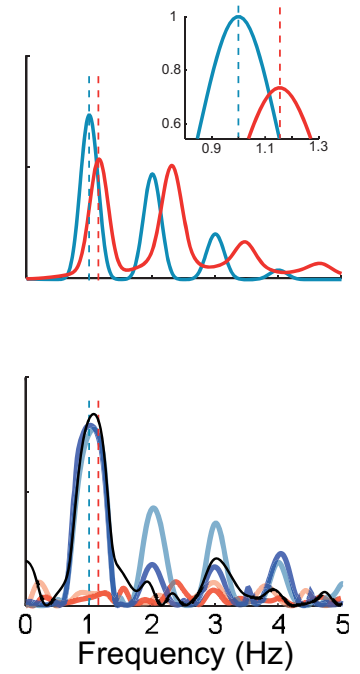

B

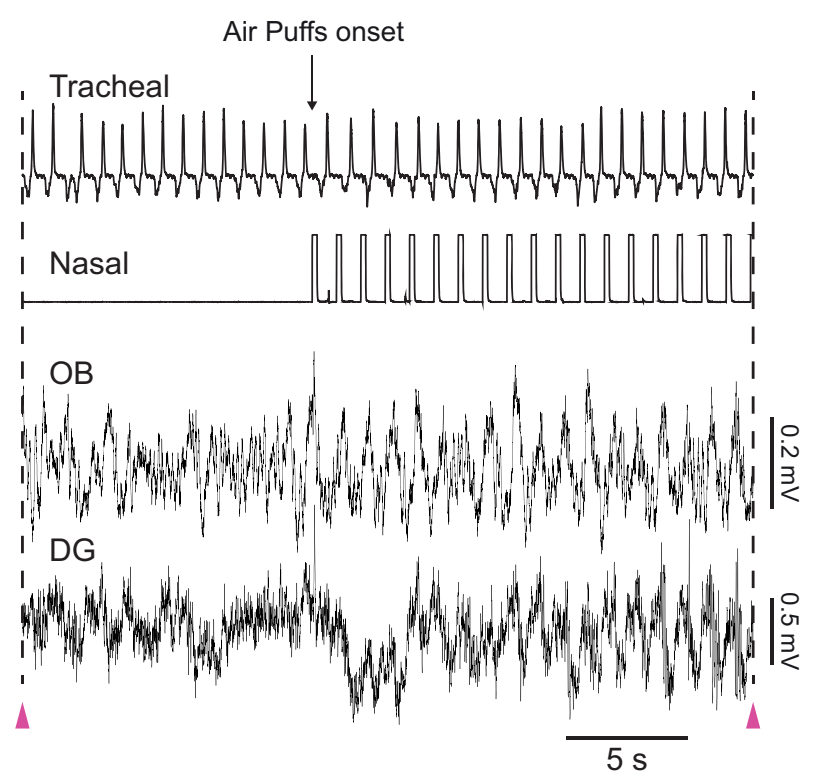

Tracheal Resp.

Tracheal Resp. + Air Puff

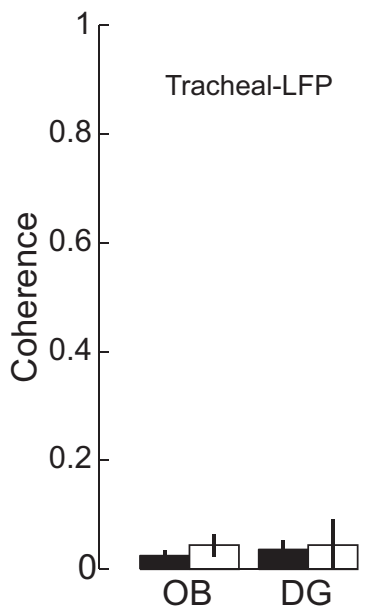

OB-DG
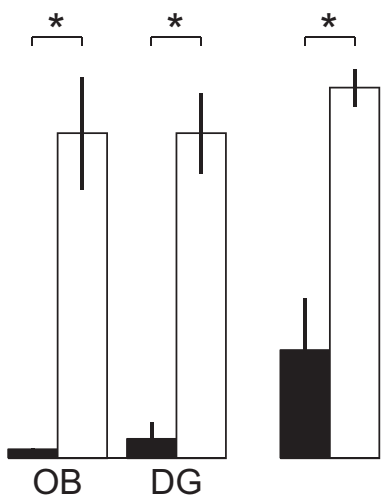

Figure 13. Rhythmic air-puffing into the nasal cavity restores coherent oscillations in OB and DG in tracheotomized rats. $A$, Time-frequency power analysis of tracheal pressure, nasal pressure, and LFP signals recorded from a urethane-anesthetized rat breathing through tracheostomy. Air puffing into the nasal cavity at $1 \mathrm{~Hz}$ drives an oscillatory LFP activity in $0 \mathrm{~B}$ and DG at the same frequency. $\boldsymbol{B}$, Raw signals for the period indicated by the purple arrowheads in $\boldsymbol{A}$. $\boldsymbol{C}$, Left, Power spectra of tracheal and nasal pressure signals during tracheal respiration before the onset of air puffing (top). Bottom, Tracheal-LFP, nasal-LFP, and OB-DG coherence spectra for the same period. Right, Similar as before, but during tracheal respiration along with air-puffing into the nasal cavity. There is high nasal-LFP and OB-DG coherence at the frequency of air puffs $(1 \mathrm{~Hz})$ and no LFP coherence with the tracheal pressure signal. $D$, Mean ( \pm SEM) tracheal-LFP, nasal-LFP, and OB-DG coherence during tracheal respiration alone (black bars) and during tracheal respiration along with air puffs (white bars) $\left(n=3\right.$ rats). ${ }^{*} p<0.05$ (paired $t$ tests).

work and others (Kopell et al., 2010; Tort et al., 2010) indicate not to be ideal (not to mention that a frequency-based definition of brain oscillations often leads to misleading interpretations when directly translating animal research to human EEG). In this case, different laboratories ended by calling "slow oscillations" different oscillatory phenomena. The "slow oscillations" in Fontanini et al. (2003) and Yanovsky et al. (2014) actually correspond to a respiration-coupled rhythm, whereas the "slow oscillations" described by the Dickson laboratory corresponds to up-and-down transitions seen in deep sleep and anesthesia.

Theta oscillations are well known to be generated in the hippocampus (Buzsáki et al., 1986; Brankačk et al., 1993). In addition, here we found that SO and HRR are also apparent in hippocampal CSD signals (Fig. 11). Because CSD is considered to eliminate volume conducted potentials (Sirota et al., 2003), these results suggest that $\mathrm{SO}$ and HRR are also generated in the hippocampus. Consistent with this, Wolansky et al. (2006) and Sharma et al. (2010) concluded that hippocampal SO has a local generator. Their conclusion is due to the fact that (1) SO has a local maximum at the hippocampal fissure, (2) hippocampal SO exhibits a small $\left(20^{\circ}\right)$ but significant phase shift across the hippocampus, (3) CSD analysis reveals sinks and sources within the hippocampus, and (4) hippocampal SO modulates local multiunit activity. Similarly, our results are also consistent with Yanovsky et al. (2014), who showed HRR to be generated in the DG. 
It should be noted that, in deep anesthesia, $\mathrm{SO}$ can often dominate LFP activity not only in the neocortex but also in the hippocampus and $\mathrm{OB}$, and the respiration-coupled rhythm may not be observed even in OB (Fig. 4). This implies that the existence of a power peak at the same frequency as respiration is not sufficient to assume the existence of an LFP rhythm coupled to respiration (Fig. 5A). Instead, our results suggest that (H)RR can only be confidently assessed when nasal respiration is also simultaneously recorded; in particular, the use of OB LFPs as a proxy for assessing respiratory activity may be misleading in some cases.

In our experiments, HRR vanished with tracheotomy, which strongly suggests that HRR is not driven by a respiratory central pattern generator in the brainstem but by a reafferent signal. Accordingly, in tracheotomized animals, we could restore rhythmic hippocampal activity locked to nasal airflow by delivering air puffs into the nasal cavity. The rhythmic entrainment of olfactory receptors in the nasal cavity may be relayed to the hippocampus by the olfactory system. Indeed, both $\mathrm{OB}$ and piriform cortex display pronounced respiration-coupled rhythmicity (Adrian, 1942; Fontanini et al., 2003). Moreover, both areas project to the entorhinal cortex (Beckstead, 1978; Wilson and Steward, 1978; Schwerdtfeger et al., 1990), which is the major sensory relay to the hippocampus (Steward and Scoville, 1976). Also supporting that the olfactory system conveys HRR to the hippocampus through the entorhinal cortex, electrical stimulation of LOT (axons from $\mathrm{OB}$ ) and PP (entorhinal axons projecting to DG) evoked hippocampal responses that had identical laminar profiles to HRR.

Ito et al. (2014) have demonstrated the existence of respiration-coupled oscillations in the whisker barrel cortex of both anesthetized and awake mice. Consistently, a recent work has shown that HRR also exists in awake mice (Nguyen Chi et al., 2016). Similarly to HRR, the respiration-coupled oscillations in the barrel cortex also depended on nasal airflow, for they were abolished with tracheotomy (Ito et al., 2014). These results show that the $\mathrm{OB}$ is capable of imposing its rhythmicity onto primary sensory areas of the neocortex. In addition, here we have found that respiration-coupled oscillations can also be detected in another neocortical region, namely, the medial prefrontal cortex (Fig. 6B). Together, these results suggest that the respiratory cycle may constitute a global signal for neuronal communication across multiple brain regions. Of note, although $\mathrm{SO}$ was not described by Ito et al. (2014), up-and-down states also occur in the barrel cortex during slow-wave sleep and anesthesia (Petersen et al., 2003). In this sense, similarly to what we reported here for the hippocampus and medial prefrontal cortex, it is quite possible that primary neocortical areas also exhibit different types of lowfrequency oscillations.

In conclusion, the bulk of our results solve previous inconsistencies in the literature regarding the entrainment of lowfrequency oscillations to the respiratory cycle, and, in agreement with previous work (Kepecs et al., 2006; Shusterman et al., 2011; Smear et al., 2011; Haddad et al., 2013; Ito et al., 2014), suggest that respiration-coupled network activity may play a role in the transfer of information between olfactory and higher-order brain regions.

\section{References}

Adrian ED (1942) Olfactory reactions in the brain of the hedgehog. J Physiol 100:459-473. CrossRef Medline

Beckstead RM (1978) Afferent connections of the entorhinal area in the rat as demonstrated by retrograde cell-labeling with horseradish peroxidase. Brain Res 152:249-264. CrossRef Medline

Brankačk J, Stewart M, Fox SE (1993) Current source density analysis of the hippocampal theta rhythm: associated sustained potentials and candidate synaptic generators. Brain Res 615:310-327. CrossRef Medline

Burwell RD, Witter MP, Amaral DG (1995) Perirhinal and postrhinal cortices of the rat: a review of the neuroanatomical literature and comparison with findings from the monkey brain. Hippocampus 5:390-408. CrossRef Medline

Buzsáki G, Draguhn A (2004) Neuronal oscillations in cortical networks. Science 304:1926-1929. CrossRef Medline

Buzsáki G, Czopf J, Kondákor I, Kellényi L (1986) Laminar distribution of hippocampal rhythmic slow activity (RSA) in the behaving rat: currentsource density analysis, effects of urethane and atropine. Brain Res 365: 125-137. CrossRef Medline

Canning KJ, Leung LS (1997) Lateral entorhinal, perirhinal, and amygdalaentorhinal transition projections to hippocampal CA1 and dentate gyrus in the rat: a current source density study. Hippocampus 7:643-655. CrossRef Medline

Canto CB, Wouterlood FG, Witter MP (2008) What does the anatomical organization of the entorhinal cortex tell us? Neural Plast 381243.

Clement EA, Richard A, Thwaites M, Ailon J, Peters S, Dickson CT (2008) Cyclic and sleep-like spontaneous alternations of brain state under urethane anaesthesia. PLoS One 3:e2004. CrossRef Medline

Colgin LL, Denninger T, Fyhn M, Hafting T, Bonnevie T, Jensen O, Moser MB, Moser EI (2009) Frequency of gamma oscillations routes flow of information in the hippocampus. Nature 462:353-357. CrossRef Medline

de Curtis M, Paré D, Llinás RR (1991) The electrophysiology of the olfactory-hippocampal circuit in the isolated and perfused adult mammalian brain in vitro. Hippocampus 1:341-354. CrossRef Medline

Delorme A, Makeig S (2004) EEGLAB: an open source toolbox for analysis of single-trial EEG dynamics including independent component analysis. J Neurosci Methods 134:9-21. CrossRef Medline

Feldman JL, Del Negro CA (2006) Looking for inspiration: new perspectives on respiratory rhythm. Nat Rev Neurosci 7:232-242. CrossRef Medline

Fontanini A, Bower JM (2005) Variable coupling between olfactory system activity and respiration in ketamine/xylazine anesthetized rats. J Neurophysiol 93:3573-3581. CrossRef Medline

Fontanini A, Bower JM (2006) Slow-waves in the olfactory system: an olfactory perspective on cortical rhythms. Trends Neurosci 29:429-437. CrossRef Medline

Fontanini A, Spano P, Bower JM (2003) Ketamine-xylazine-induced slow $(<1.5 \mathrm{~Hz})$ oscillations in the rat piriform (olfactory) cortex are functionally correlated with respiration. J Neurosci 23:7993-8001. Medline

Grosmaitre X, Santarelli LC, Tan J, Luo M, Ma M (2007) Dual functions of mammalian olfactory sensory neurons as odor detectors and mechanical sensors. Nat Neurosci 10:348-354. CrossRef Medline

Haddad R, Lanjuin A, Madisen L, Zeng H, Murthy VN, Uchida N (2013) Olfactory cortical neurons read out a relative time code in the olfactory bulb. Nat Neurosci 16:949-957. CrossRef Medline

Ito J, Roy S, Liu Y, Cao Y, Fletcher M, Lu L, Boughter JD, Grün S, Heck DH (2014) Whisker barrel cortex $\delta$ oscillations and gamma power in the awake mouse are linked to respiration. Nat Commun 5:3572. CrossRef Medline

Kepecs A, Uchida N, Mainen ZF (2006) The sniff as a unit of olfactory processing. Chem Senses 31:167-179. CrossRef Medline

Kleinfeld D, Deschênes M, Wang F, Moore JD (2014) More than a rhythm of life: breathing as a binder of orofacial sensation. Nat Neurosci 17:647651. CrossRef Medline

Kopell N, Kramer MA, Malerba P, Whittington MA (2010) Are different rhythms good for different functions? Front Hum Neurosci 4:187. CrossRef Medline

Lockmann AL, Belchior H (2014) New insights into the role of respiratory inputs in hippocampal oscillations. J Neurosci 34:9473-9475. CrossRef Medline

Nguyen Chi V, Müller C, Wolfenstetter T, Yanovsky Y, Draguhn A, Tort AB, Brankačk J (2016) Hippocampal respiration-driven rhythm distinct from theta oscillations in awake mice. J Neurosci 36:162-177. CrossRef Medline

Pagliardini S, Greer JJ, Funk GD, Dickson CT (2012) State-dependent modulation of breathing in urethane-anesthetized rats. J Neurosci 32:1125911270. CrossRef Medline

Paxinos G, Watson C (2004) The rat brain in stereotaxic coordinates: the new coronal set. San Diego: Academic.

Petersen CC, Hahn TT, Mehta M, Grinvald A, Sakmann B (2003) Interac- 
tion of sensory responses with spontaneous depolarization in layer $2 / 3$ barrel cortex. Proc Natl Acad Sci U S A 100:13638-13643. CrossRef Medline

Schall KP, Kerber J, Dickson CT (2008) Rhythmic constraints on hippocampal processing: state and phase-related fluctuations of synaptic excitability during theta and the slow oscillation. J Neurophysiol 99: 888-899. CrossRef Medline

Scheffer-Teixeira R, Belchior H, Caixeta FV, Souza BC, Ribeiro S, Tort AB (2012) Theta phase modulates multiple layer-specific oscillations in the CA1 region. Cereb Cortex 22:2404-2414. CrossRef Medline

Schomburg EW, Fernández-Ruiz A, Mizuseki K, Berényi A, Anastassiou CA, Koch C, Buzsáki G (2014) Theta phase segregation of input-specific gamma patterns in entorhinal-hippocampal networks. Neuron 84:470485. CrossRef Medline

Schwerdtfeger WK, Buhl EH, Germroth P (1990) Disynaptic olfactory input to the hippocampus mediated by stellate cells in the entorhinal cortex. J Comp Neurol 292:163-177. CrossRef Medline

Sharma AV, Wolansky T, Dickson CT (2010) A comparison of sleeplike slow oscillations in the hippocampus under ketamine and urethane anesthesia. J Neurophysiol 104:932-939. CrossRef Medline

Shusterman R, Smear MC, Koulakov AA, Rinberg D (2011) Precise olfactory responses tile the sniff cycle. Nat Neurosci 14:1039-1044. CrossRef Medline

Sirota A, Csicsvari J, Buhl D, Buzsáki G (2003) Communication between neocortex and hippocampus during sleep in rodents. Proc Natl Acad Sci U S A 100:2065-2069. CrossRef Medline

Smear M, Shusterman R, O'Connor R, Bozza T, Rinberg D (2011) Perception of sniff phase in mouse olfaction. Nature 479:397-400. CrossRef Medline

Steriade M, Nuñez A, Amzica F (1993a) Intracellular analysis of relations between the slow $(<1 \mathrm{~Hz})$ neocortical oscillation and other sleep rhythms of the electroencephalogram. J Neurosci 13:3266-3283. Medline
Steriade M, Nuñez A, Amzica F (1993b) A novel slow ( $<1 \mathrm{~Hz})$ oscillation of neocortical neurons in vivo: depolarizing and hyperpolarizing components. J Neurosci 13:3252-3265. Medline

Steward O, Scoville SA (1976) Cells of origin of entorhinal cortical afferents to the hippocampus and fascia dentata of the rat. J Comp Neurol 169: 347-370. CrossRef Medline

Tort AB, Fontanini A, Kramer MA, Jones-Lush LM, Kopell NJ, Katz DB (2010) Cortical networks produce three distinct $7-12 \mathrm{~Hz}$ rhythms during single sensory responses in the awake rat. J Neurosci 30:4315-4324. CrossRef Medline

Vanderwolf CH (1992) Hippocampal activity, olfaction, and sniffing: an olfactory input to the dentate gyrus. Brain Res 593:197-208. CrossRef Medline

Verhagen JV, Wesson DW, Netoff TI, White JA, Wachowiak M (2007) Sniffing controls an adaptive filter of sensory input to the olfactory bulb. Nat Neurosci 10:631-639. CrossRef Medline

Viczko J, Sharma AV, Pagliardini S, Wolansky T, Dickson CT (2014) Lack of respiratory coupling with neocortical and hippocampal slow oscillations. J Neurosci 34:3937-3946. CrossRef Medline

Wachowiak M (2011) All in a sniff: olfaction as a model for active sensing. Neuron 71:962-973. CrossRef Medline

Wilson RC, Steward O (1978) Polysynaptic activation of the dentate gyrus of the hippocampal formation: an olfactory input via the lateral entorhinal cortex. Exp Brain Res 33:523-534. Medline

Wolansky T, Clement EA, Peters SR, Palczak MA, Dickson CT (2006) Hippocampal slow oscillation: a novel EEG state and its coordination with ongoing neocortical activity. J Neurosci 26:6213-6229. CrossRef Medline

Yanovsky Y, Ciatipis M, Draguhn A, Tort AB, Brankačk J (2014) Slow oscillations in the mouse hippocampus entrained by nasal respiration. J Neurosci 34:5949-5964. CrossRef Medline 